\title{
SYNCHRONIZATION OF KURAMOTO OSCILLATORS: INVERSE TAYLOR EXPANSIONS*
}

\author{
SABER JAFARPOUR ${ }^{\dagger}$, ELIZABETH Y. HUANG ${ }^{\ddagger}$, AND FRANCESCO BULLO $^{\S}$
}

\begin{abstract}
Synchronization in networks of coupled oscillators is a widely studied topic with extensive scientific and engineering applications. In this paper, we study the frequency synchronization problem for networks of Kuramoto oscillators with arbitrary topology and heterogeneous edge weights. We propose a novel equivalent transcription for the equilibrium synchronization equation. Using this transcription, we develop a power series expansion to compute the synchronized solution of the Kuramoto model as well as a sufficient condition for the strong convergence of this series expansion. Truncating the power series provides (i) an efficient approximation scheme for computing the synchronized solution, and (ii) a simple-to-check, statistically-correct hierarchy of increasingly accurate synchronization tests. This hierarchy of tests provides a theoretical foundation for and generalizes the best-known approximate synchronization test in the literature. Our numerical experiments illustrate the accuracy and the computational efficiency of the truncated series approximation compared to existing iterative methods and existing synchronization tests.
\end{abstract}

Key words. Kuramoto oscillators, frequency synchronization, synchronization manifold, Taylor series, power networks

AMS subject classifications. 34D06, 34C15, 93D20, 37C25, 37M20

1. Introduction. Collective synchronization is an interesting behaviour which lies at the heart of various natural phenomena. The celebrated Kuramoto model [22] is one of the simplest models for studying synchronization in a network of coupled oscillators. Kuramoto model has been successfully used to model the synchronization behaviour of a wide range of physical, chemical, and biological system [2]. Examples include the power grids [15, 10], automated vehicle coordination [21, 31], pacemakers in heart [42], clock synchronization [32], and neural networks [13]; see also [6, Chapter 13] for additional examples. One of the most interesting types of synchronization is frequency synchronization, where all oscillators reach the same rotational frequency with possibly different phases. It is well-known that the Kuramoto model can exhibit a transition from incoherence to frequency synchronization. For many applications, such as power networks, it is important to have an accurate estimate of this transition to synchronization. This is essential, particularly as the grid is being pushed closer to its maximum capacity due to increases in the load demand and penetration of renewable energy units. Finding sharp conditions to determine when this transition happens continues to be a challenging as well as a critical problem.

Literature Review. The problem of finding conditions for existence of a stable synchronized solution for the Kuramoto model of coupled oscillators has been studied extensively in the literature. For complete graphs with homogeneous weights, the order parameter is used to implicitly determine the exact critical coupling needed for a synchronized solution [3, 25, 39]. For acyclic graphs with heterogeneous weights, a necessary and sufficient condition is developed for synchronization of the heteroge-

${ }^{*}$ This work was supported in part by the U.S. Department of Energy (DOE) Solar Energy Technologies Office under Contract No. DE-EE0000-1583.

${ }^{\dagger}$ Center for Control, Dynamical Systems, and Computation, University of California, Santa Barbara (saber.jafarpour@engineering.ucsb.edu).

${ }^{\ddagger}$ Center for Control, Dynamical Systems, and Computation, University of California, Santa Barbara (eyhuang@engineering.ucsb.edu).

$\S$ Department of Mechanical Engineering and Center for Control, Dynamical Systems, and Computation, University of California, Santa Barbara (bullo@engineering.ucsb.edu) 
neous Kuramoto model [12]. In addition, Lyapunov analysis applied to the complete graph is used in [8] to give a sufficient condition and in [9] to give an explicit necessary and sufficient condition for existence of a synchronized solution. However for general topology graphs, such a complete characterization of frequency synchronization does not exist. For general graphs with heterogeneous weights, several necessary conditions and sufficient conditions for existence of stable synchronized solution have been reported in the literature. [36] requires sufficiently large nodal degrees relative to the natural frequencies, [4] uses the cutset in the graph, and [18] states that the algebraic connectivity must be sufficiently large compared to the difference in natural frequencies of connected oscillators. Recently, a novel cutset projection operator has been introduced to rigorously prove a simple-to-check, sufficient condition for synchronization of Kuramoto model [19]. Using numerous simulations, it is shown that this new sufficient condition scales better to large networks [19].

Despite these deep results in the literature, the existing synchronization conditions usually provide conservative estimates for the synchronization threshold. In an effort to come closer to finding the exact synchronization threshold, [12] and [17] introduce a statistically accurate approximate test for synchronization that depends on the network parameters and topology derived from the linearized Kuramoto map and the converging power series expansion of the phase angles of the Kuramoto oscillators, respectively.

If existence of a frequency synchronized solution can be guaranteed, then the next step is computing the synchronized solutions. A common method to approximate the solution is to linearize the equations. This will result in studying the equations of the form $\omega=L \theta$, where $L$ is the Laplacian matrix of the network [23, 33, 35]. The angles $\theta$ can be approximately solved very efficiently, even for extremely large, sparse graphs [40, Theorem 3.1]. However, when phase differences of the oscillators are large, this linear approximation is not very accurate.

In order to compute the synchronization manifold of the nonlinear Kuramoto equations, one can employ iterative numerical algorithms such as Newton-Raphson or Gauss-Seidel [34, 37, 14]. Unfortunately, these algorithms do not guarantee convergence to the synchronized solutions and failure of these algorithms could be due to numerical instability, an initialization issue, or non-existence of the solution. Another approach is to use numerical polynomial homotopy continuation (NPHC). It is guaranteed that NPHC will find all stable and unstable manifolds of the Kuramoto model, but this method is not computationally tractable for large networks; [24] uses NPHC to study the homogeneous Kuramoto model for particular graph topologies with up to 18 nodes. Finally, [41] gives an approximate analytical solution for stable synchronization manifolds using the order parameter. However, this approximation scheme is only applicable to the uniform-weight Kuramoto model with all-to-all connections.

A wide range of methods for finding synchronized solutions of the Kuramoto model stem from the power network literature, where different techniques are used to find the solutions of the $\mathrm{AC}$ power flow equations. Here, we only review two of these approaches. The first approach is called Holomorphic Embedding Load-Flow Method (HELM) and has been proposed to find all the solutions of power flow equations [38]. While HELM is based on advanced results and concepts from complex analysis, its numerical implementation is recursive and straightforward [38, 30]. However, HELM is reported to be much slower than the Newton-Raphson methods [30]. The second approach is the optimization approach, whereas an optimal power flow problem (OPF) is used to solve for the $\mathrm{AC}$ power flow equations. The OPF problems have been studied extensively in the power network literature, e.g., see [27, 28, 26]. Thus, one can use the 
numerical algorithms for the optimization problem to find the synchronized solution of the Kuramoto model. Unfortunately, due to the non-convex nature of the OPFs, these algorithms usually result in an approximation of the synchronized solution.

Contribution. The contributions of this paper are both theoretical and computational. From a theoretical viewpoint, first, we review important properties of the Kuramoto model of coupled oscillators and, as a minor contribution, we provide a rigorous proof for the following well-known folk theorem: frequency synchronization is equivalent with the existence of a stable synchronization manifold (see [25] and [39] for statement of this result without proof). Second, by introducing the notions of edge vectors and flow vectors in graphs, we propose four equivalent transcriptions for the synchronization manifold of the Kuramoto model: node, flow, constrained edge, and unconstrained edge balance equations. While the first three formulations have already been studied in the literature (see [12] and [19]), the unconstrained edge balance equations provide a novel important characterization of the synchronization manifold. Our main technical results are (1) a sufficient condition for existence of a unique solution for unconstrained edge balance equations and (2) a recursive expression for each term of the Taylor series expansion for this solution of the unconstrained edge balance equations. Additionally, we prove that, if our simple-to-check sufficient condition is satisfied, then the Taylor series expansion for the solution converges strongly. We also provide an algorithm to symbolically compute all terms of the expansion. Third and final, using the one-to-one correspondence between solutions of the unconstrained edge balance equations and synchronized solutions of the Kuramoto model, we propose a power series expansion for the synchronized solutions of the Kuramoto model and an estimate on the region of convergence of the power series.

From a computational viewpoint, first, we propose a method to approximate the synchronization manifold of the Kuramoto model using the truncated power series. We present several numerical experiments using IEEE test cases and random graphs to illustrate (1) the accuracy of the truncated series and (2) the computational efficiency of the new methods for computing the synchronization manifold. We show that the seventh order approximate method has low absolute error when applied to IEEE test cases with weakly coupled oscillators. The truncated series, up to the seventh order, have comparable computational efficiency to Newton-Raphson when solving for solutions with static graph topology and multiple natural frequencies, or power injections. Second, based on our novel power series approach, we propose a hierarchy of approximate tests for synchronization of the Kuramoto model; our approach provides a theoretical basis for and generalize the state-of-the-art approximate synchronization test in the literature [12]. With numerical analysis, we verify the accuracy of our family of approximate tests for several random graphs and numerous IEEE test cases. In each of these cases, we show that our new approximate tests are a significant improvement compared to the best-known approximate condition given in [12].

Finally, we compare this paper with our preliminary conference article [17]. In short, this paper presents a substantially more complete and comprehensive treatment of the power series approach to synchronization of Kuramoto oscillators. Specifically, while [17] presents a power series expansion for nodal phase angles, this paper develops a novel power series expansion for the flows in the network. Using the Banach FixedPoint Theorem, we provide an estimate on the domain of convergence of the power series which is substantially larger than the estimates given in [17]. Moreover, our numerical analysis shows that the hierarchy of approximate synchronization tests 
obtained by truncating this power series is more accurate than the estimate tests proposed in [17].

Paper organization. In Section 2, we give preliminaries and notation used in the paper. In Section 3-4 we review the Kuramoto model, frequency synchronization, and give several equivalent formulations of the algebraic Kuramoto equation. Sections 5 and 6 contain the paper's main theoretical results and a family of approximate synchronization tests. Finally, Section 7 contains numerical experiments analyzing the approximate synchronization tests and efficiency of computation methods for the synchronization manifold.

\section{Preliminaries and notation.}

Vectors and functions. Let $\mathbb{Z}_{>0}, \mathbb{R}^{n}$, and $\mathbb{C}^{n}$ denote the set of non-negative integers, the $n$-dimensional real Euclidean space, and the $n$-dimensional complex Eu-

clidean space, respectively. For $n \in \mathbb{Z}_{\geq 0}$, let $n ! !=\prod_{k=0}^{\left\lceil\frac{n}{2}\right\rceil-1}(n-2 k)$ denote the double factorial. For $r>0$ and $\mathbf{x} \in \mathbb{R}^{n}$, the real polydisk with center $\mathbf{x}$ and radius $r$ is

$$
\mathrm{D}_{n}(\mathbf{x}, r)=\left\{\mathbf{y} \in \mathbb{R}^{n} \mid\|\mathbf{x}-\mathbf{y}\|_{\infty} \leq r\right\} .
$$

Similarly, for $r>0$ and $\mathbf{z} \in \mathbb{C}^{n}$, the complex polydisk with center $\mathbf{z}$ and radius $r$ is

$$
\mathrm{D}_{n}^{\mathbb{C}}(\mathbf{z}, r)=\left\{\mathbf{w} \in \mathbb{C}^{n} \mid\|\mathbf{z}-\mathbf{w}\|_{\infty} \leq r\right\} .
$$

Let $\mathbb{1}_{n}$ and $\mathbb{O}_{n}$ be $n$-dimensional column vectors of ones and zeros respectively. For $\mathbf{x}=\left(x_{1}, \ldots, x_{n}\right)^{\top} \in \mathbb{C}^{n}$, let $\sin (\mathbf{x})=\left(\sin \left(x_{1}\right), \ldots, \sin \left(x_{n}\right)\right)^{\top}$ and $\operatorname{diag}(\mathbf{x})$ be the $n \times n$ diagonal matrix with $(\operatorname{diag}(\mathbf{x}))_{i i}=x_{i}$, for every $i \in\{1, \ldots, n\}$. For $\mathbf{x}=$ $\left(x_{1}, \ldots, x_{n}\right)^{\top} \in \mathbb{C}^{n}$ with $\|\mathbf{x}\|_{\infty} \leq 1$, let $\arcsin (\mathbf{x})=\left(\arcsin \left(x_{1}\right), \ldots, \arcsin \left(x_{n}\right)\right)^{\top}$, where

$$
\arcsin (r)=\sum_{i=0}^{\infty} \frac{(2 i-1) ! !}{(2 i) ! !(2 i+1)} r^{2 i+1} .
$$

For every $n \in \mathbb{N}$, we denote the $n$-torus by $\mathbb{T}^{n}$. For every $s \in[0,2 \pi)$, the clockwise rotation of $\theta \in \mathbb{T}^{n}$ by the angle $s$ is the function $\operatorname{rot}_{s}: \mathbb{T}^{n} \rightarrow \mathbb{T}^{n}$ defined by

$$
\operatorname{rot}_{s}(\theta)=\left(\theta_{1}+s, \ldots, \theta_{n}+s\right)^{\top}, \quad \text { for } \theta \in \mathbb{T}^{n}
$$

Using the rotation function, one defines an equivalence relation $\sim$ on the $n$-torus $\mathbb{T}^{n}$ as follows: For every two points $\theta, \eta \in \mathbb{T}^{n}$, we say $\theta \sim \eta$ if there exists $s \in[0,2 \pi)$ such that $\theta=\operatorname{rot}_{s}(\eta)$. For every $\theta \in \mathbb{T}^{n}$, the equivalence class of $\theta$ is denoted by $[\theta]=\left\{\operatorname{rot}_{s}(\theta) \mid s \in[0,2 \pi)\right\}$. The quotient space of $\mathbb{T}^{n}$ under the equivalence relation $\sim$ is denoted by $\left[\mathbb{T}^{n}\right]$.

Algebraic graph theory. Let $G$ be a weighted undirected connected graph with the node set $\mathcal{N}=\{1, \ldots, n\}$ and the edge set $\mathcal{E} \subseteq \mathcal{N} \times \mathcal{N}$ with $m$ elements. We assume that $G$ has no self-loops and the weights of the edges are described by the nonnegative, symmetric adjacency matrix $A \in \mathbb{R}^{n \times n}$. The Laplacian matrix of the graph $G$ is $L=\operatorname{diag}\left(A \mathbb{1}_{n}\right)-A \in \mathbb{R}^{n \times n}$. Define the diagonal edge weight matrix by $\mathcal{A}=\operatorname{diag}\left(a_{i j \in \mathcal{E}}\right) \in \mathbb{R}^{m \times m}$. It is known that the Laplacian is $L=B \mathcal{A} B^{\top}$. Since $L$ is singular, we use the Moore-Penrose pseudoinverse $L^{\dagger}$ which has the following properties: $L L^{\dagger} L=L, L^{\dagger} L L^{\dagger}=L^{\dagger}, L^{\dagger} L=\left(L^{\dagger} L\right)^{\top}$, and $L L^{\dagger}=\left(L L^{\dagger}\right)^{\top}$. In addition, 
for a connected graph $L^{\dagger} L=L L^{\dagger}=I_{n}-\frac{1}{n} \mathbb{1}_{n}^{\top} \mathbb{1}_{n}$. The weighted cutset projection matrix $\mathcal{P}_{\text {cut }}$ is the oblique projection onto $\operatorname{Img}\left(B^{\top}\right)$ parallel to $\operatorname{Ker}(B \mathcal{A})$ given by

$$
\mathcal{P}_{\text {cut }}=B^{\top} L^{\dagger} B \mathcal{A} \text {. }
$$

The weighted cutset projection matrix $\mathcal{P}_{\text {cut }}$ is idempotent, and 0 and 1 are its eigenvalues with algebraic (and geometric) multiplicity $m-n+1$ and $n-1$, respectively. Additional properties of $\mathcal{P}_{\text {cut }}$ are in [19, Theorem 5]. Similarly, the weighted cycle projection matrix $\mathcal{P}_{\text {cyc }}$ is the oblique projection onto $\operatorname{Ker}(B \mathcal{A})$ parallel to $\operatorname{Img}\left(B^{\top}\right)$ given by

$$
\mathcal{P}_{\text {cyc }}=I_{m}-B^{\top} L^{\dagger} B \mathcal{A} \text {. }
$$

Analytic functions and power series. A multi-index $\nu$ is a member of $\left(\mathbb{Z}_{>0}\right)^{n}$. For every $x \in \mathbb{C}^{n}$, we define $x^{\nu}=x_{1}^{\nu_{1}} x_{2}^{\nu_{2}} \ldots x_{n}^{\nu_{n}}$. For $x_{0} \in \mathbb{C}^{n}$, the formal expression

$$
\sum_{\nu \in\left(\mathbb{Z}_{>0}\right)^{n}} a_{\nu}\left(x-x_{0}\right)^{\nu}
$$

where $a_{\nu} \in \mathbb{C}$, for every $\nu \in\left(\mathbb{Z}_{>0}\right)^{n}$ is called a formal power series around point $x_{0}$. The power series $\sum_{\nu \in\left(\mathbb{Z}_{>0}\right)^{n}} a_{\nu}\left(x-x_{0}\right)^{\nu}$ converges strongly at point $x$ if all rearrangement of the terms of the series $\sum_{\nu} a_{\nu}\left(x-x_{0}\right)^{\nu}$ converges. For every $x_{0} \in \mathbb{C}^{n}$, the domain of convergence of (1) around $x_{0}$ is defined as the set $\mathcal{C}_{x_{0}}$ of all points $x \in \mathbb{C}^{n}$ such that the power series $\sum_{\nu} a_{\nu}\left(x-x_{0}\right)^{\nu}$ converges strongly at point $x$. While for $n=1$, one can show that the domain of convergence is an open interval around $x_{0}$, for $n>1$ the domain of convergence of a power series is not necessarily an open poly-disk around $x_{0}$. An open set $\Omega \subset \mathbb{C}^{n}$ is a Reinhardt domain if, for every $\left(z_{1}, \ldots, z_{n}\right)^{\top} \in \Omega$ and every $\left(\theta_{1}, \ldots, \theta_{n}\right)^{\top} \in \mathbb{T}^{n}$, we have $\left(e^{i \theta_{1}} z_{1}, \ldots, e^{i \theta_{n}} z_{n}\right)^{\top} \in \Omega$. The Reinhardt domains can be considered as the generalization of the disks on the complex plane to higher dimensions.

3. The heterogeneous Kuramoto model. The Kuramoto model is a system of $n$ oscillators, where each oscillator has a natural frequency $\omega_{i} \in \mathbb{R}$ and its state is represented by a phase angle $\theta_{i} \in \mathbb{S}^{1}$. The interconnection of these oscillators are described using a weighted undirected connected graph $G$, with nodes $\mathcal{N}=\{1, \ldots, n\}$, edges $\mathcal{E} \subseteq \mathcal{N} \times \mathcal{N}$, and positive weights $a_{i j}=a_{j i}>0$. The dynamics for the heterogeneous Kuramoto model is given by:

$$
\dot{\theta}_{i}=\omega_{i}-\sum_{j=1}^{n} a_{i j} \sin \left(\theta_{i}-\theta_{j}\right), \quad \text { for } i \in\{1, \ldots, n\} .
$$

In matrix language, one can write this differential equations as:

$$
\dot{\theta}=\omega-B \mathcal{A} \sin \left(B^{\top} \theta\right),
$$

where $\theta=\left(\theta_{1}, \theta_{2}, \ldots, \theta_{n}\right)^{\top} \in \mathbb{T}^{n}$ is the phase vector, $\omega=\left(\omega_{1}, \omega_{2}, \ldots, \omega_{n}\right)^{\top} \in \mathbb{R}^{n}$ is the natural frequency vector, and $B$ is the incidence matrix for the graph $G$. One can show that if $\theta: \mathbb{R}_{\geq 0} \rightarrow \mathbb{T}^{n}$ is a solution for the Kuramoto model (3) then, for every $s \in[0,2 \pi)$, the curve $\operatorname{rot}_{s}(\theta): \mathbb{R}_{\geq 0} \rightarrow \mathbb{T}^{n}$ is also a solution of (3). Therefore, for the rest of this paper, we consider the state space of the Kuramoto model (3) to be [ $\left.\mathbb{T}^{n}\right]$. 
Definition 1 (Frequency synchronization). A solution $\theta: \mathbb{R}_{\geq 0} \rightarrow\left[\mathbb{T}^{n}\right]$ of the coupled oscillator model (3) achieves frequency synchronization if there exists a frequency $\omega_{\text {syn }} \in \mathbb{R}$ such that

$$
\lim _{t \rightarrow \infty} \dot{\theta}(t)=\omega_{\mathrm{syn}} \mathbb{1}_{n}
$$

By summing all the equations in (2), one can show that if a solution of (3) achieves frequency synchronization then $\omega_{\text {syn }}=\frac{1}{n} \sum_{i=1}^{n} \omega_{i}$. Therefore, without loss of generality, we can assume that in the Kuramoto model (3), we have $\omega \in \mathbb{1}_{n}^{\perp}$ and $\omega_{\text {syn }}=0$.

DEFINITION 2 (Synchronization manifold). Let $\theta^{*}$ be a solution of the algebraic equation

$$
\omega=B \mathcal{A} \sin \left(B^{\top} \theta^{*}\right)
$$

Then $\left[\theta^{*}\right]$ is called a synchronization manifold for the Kuramoto model (3).

The following theorem reduces the problem of local frequency synchronization in the Kuramoto model (3) to the existence of a solution for the algebraic equations (4).

THEOREM 3 (Characterization of frequency synchronization). For the heterogeneous Kuramoto model (3) on graph $G$, the following statements are equivalent:

(i) there exists an open set $U \in\left[\mathbb{T}^{n}\right]$ such that every solution of the Kuramoto model (3) starting in set $U$ achieves frequency synchronization;

(ii) there exists a locally asymptotically stable synchronization manifold $\left[\theta^{*}\right]$ for (3).

Additionally, if any of equivalent conditions ( $i$ ) or (ii) holds, then, for every $\theta(0) \in U$, we have $\lim _{t \rightarrow \infty}[\theta(t)]=\left[\theta^{*}\right]$.

Proof. Regarding $(i) \Longrightarrow($ ii), if the solution achieves frequency synchronization, then $\lim _{t \rightarrow \infty} \dot{\theta}_{i}(t)=0=\lim _{t \rightarrow \infty}\left(\omega_{i}-\sum_{j=1}^{n} a_{i j} \sin \left(\theta_{i}(t)-\theta_{j}(t)\right)\right)$ for all $i=\{1, \ldots, n\}$. Consider a sequence of natural numbers $k \in \mathbb{N}$ and the corresponding sequence $\theta(k)$ in $\mathbb{T}^{n}$. Since $\mathbb{T}^{n}$ is a compact metric space, it is sequentially compact $[29$, Theorem 28.2]. This means that there is a subsequence $\hat{k}$ such that $\theta_{i}(\hat{k})$ is convergent. Then $\lim _{\hat{k} \rightarrow \infty} \theta_{i}(\hat{k})$ exists and $\mathbb{D}_{n}=\omega-B \mathcal{A} \sin \left(B^{\top} \lim _{\hat{k} \rightarrow \infty} \theta(\hat{k})\right)$. Therefore $\lim _{\hat{k} \rightarrow \infty} \theta(\hat{k})$ is a synchronization manifold because it is a solution for equation (4) and is locally asymptotically stable since all solutions starting in $U$ reach $\lim _{\hat{k} \rightarrow \infty} \theta(\hat{k})$.

Regarding $(i i) \Longrightarrow(i)$, by the definition of local asymptotic stability, there exists some $\delta>0$ such that the open set $U$ is defined to be $U=\left\{\theta(0) \in\left[\mathbb{T}^{n}\right] \mid\left\|\theta(0)-\theta^{*}\right\| \leq\right.$ $\delta\}$ where $\left[\theta^{*}\right]$ is the synchronization manifold. Then for solutions starting in $U$, $\lim _{t \rightarrow \infty} \dot{\theta}(t)=\omega-B \mathcal{A} \sin \left(B^{\top} \theta^{*}\right)=0$ so $\left[\theta^{*}\right]$ is also a frequency synchronized solution for (3).

The last statement follows from the proofs of $(i) \Longrightarrow(i i)$ and $(i i) \Longrightarrow(i)$.

In many application, such as power networks, not only is it important to study the frequency synchronization of the Kuramoto oscillators but also it is essential to bound the position of the synchronization manifold $\left[\theta^{*}\right]$ due to some security constraints for the grid. An important class of security constraints are thermal constraints which are usually expressed as bounds on the geodesic distances $\left|\theta_{i}^{*}-\theta_{j}^{*}\right|$, for $i, j \in\{1, \ldots, n\}$. The geodesic distance $\left|\theta_{i}^{*}-\theta_{j}^{*}\right|$ is defined as the minimum of the clockwise and counterclockwise arc lengths between the phase angles $\theta_{i}^{*}, \theta_{j}^{*} \in \mathbb{T}^{1}$. Let $G$ be an undirected 
weighted connected graph with edge set $\mathcal{E}$ and let $\gamma \in[0, \pi)$ We define the cohesive subset $\Delta^{G}(\gamma) \subseteq\left[\mathbb{T}^{n}\right]$ by

$$
\Delta^{G}(\gamma)=\left\{[\theta] \in\left[\mathbb{T}^{n}\right]|| \theta_{i}-\theta_{j} \mid \leq \gamma, \text { for all }(i, j) \in \mathcal{E}\right\} .
$$

For every $\gamma \in[0, \pi)$, we define the embedded cohesive subset $S^{G}(\gamma) \subseteq\left[\mathbb{T}^{n}\right]$ by:

$$
S^{G}(\gamma)=\left\{[\exp (\mathrm{ix})] \mid \mathbf{x} \in B^{G}(\gamma), s \in[0,2 \pi)\right\},
$$

where $B^{G}(\gamma)=\left\{\mathbf{x} \in \mathbb{1}_{n}^{\perp} \mid\left\|B^{\top} \mathbf{x}\right\|_{\infty} \leq \gamma\right\}$. Note that, in general, we have $S^{G}(\gamma) \subseteq$ $\Delta^{G}(\gamma)$. We refer to [19] for additional properties of embedded cohesive subset. In particular, it is shown that $S^{G}(\gamma)$ is diffeomorphic with $B^{G}(\gamma)$, for every $\gamma \in\left[0, \frac{\pi}{2}\right)[19$, Theorem 8]. Using this result, in the rest of this paper we identify the set $S^{G}(\gamma)$ with $B^{G}(\gamma)$.

4. Equivalent transcriptions of the equilibrium manifold. Consider an undirected graph $G$ with vertex set $\mathcal{N}=\{1, \ldots, n\}$ and edge set $\mathcal{E} \subseteq \mathcal{N} \times \mathcal{N}$ with $|\mathcal{E}|=m$. We start by introducing three vector spaces defined by $G$ :

(i) the node space is $\mathbb{R}^{n}$; elements of this space are called node vectors;

(ii) the edge space is $\mathbb{R}^{m}$; elements of this space are called edge vectors; and

(iii) the flow vector space is $\operatorname{Img}\left(B^{\top}\right)$; elements of $\mathbb{R}^{m}$ belonging to this space are called by flow vectors.

It is easy to see that an edge vector $\mathbf{z} \in \mathbb{R}^{m}$ is a flow vector if and only if there exists a node vector $\mathbf{x} \in \mathbb{R}^{n}$ such that $\mathbf{z}=B^{\top} \mathbf{x}$.

Next, we introduce four different balance equations on an undirected graph $G$ with incidence matrix $B$, weight matrix $\mathcal{A}$, cutset projection $\mathcal{P}_{\text {cut }}$, and cycle projection $\mathcal{P}_{\text {cyc. }}$. Given a node vector $\omega \in \mathbb{1}_{n}^{\perp}$, define the shorthand flow vector $\eta=B^{\top} L^{\dagger} \omega \in$ $\operatorname{Img}\left(B^{\top}\right)$. The node balance equations in the unknown node vector $\mathbf{x} \in \mathbb{1}_{n}^{\perp}$ is

$$
\omega=B \mathcal{A} \sin \left(B^{\top} \mathbf{x}\right) .
$$

The flow balance equations in the unknown flow vector $\mathbf{z} \in \operatorname{Img}\left(B^{\top}\right)$ is

$$
\eta=\mathcal{P}_{\text {cut }} \sin (\mathbf{z}) .
$$

The constrained edge balance equations in the unknown edge vector $\psi \in \mathbb{R}^{m}$ is

$$
\left\{\begin{array}{l}
\eta=\mathcal{P}_{\text {cut }} \psi, \\
\arcsin (\psi) \in \operatorname{Img}\left(B^{\top}\right), \quad\|\psi\|_{\infty} \leq 1 .
\end{array}\right.
$$

The unconstrained edge balance equations in the unknown edge vector $\phi \in \mathbb{R}^{m}$ is

$$
\eta=\mathcal{P}_{\text {cut }} \phi+\mathcal{P}_{\text {cyc }} \arcsin (\phi), \quad\|\phi\|_{\infty} \leq 1 .
$$

We now present equivalent characterizations for synchronization manifold of the Kuramoto model (3).

THEOREM 4 (Characterization of synchronization manifold). Consider an undirected connected graph $G$ with incidence matrix $B$, weight matrix $\mathcal{A}$, cutset projection $\mathcal{P}_{\text {cut }}$, and cycle projection $\mathcal{P}_{\text {cyc. }}$. Given a node vector $\omega \in \mathbb{1}_{n}^{\perp}$, define the shorthand $\eta=B^{\top} L^{\dagger} \omega \in \operatorname{Img}\left(B^{\top}\right)$. Pick an angle $\gamma \in\left[0, \frac{\pi}{2}\right)$. Then the following statements are equivalent: 
(i) there exists a unique locally exponentially stable synchronization manifold $\mathbf{x}^{*}$ for the Kuramoto model (3) in $S^{G}(\gamma)$;

(ii) the node balance equations (5) have a unique solution $\mathbf{x}^{*}$ in $S^{G}(\gamma)$;

(iii) the flow balance equations (6) have a unique solution $\mathbf{z}^{*} \in \operatorname{Img}\left(B^{\top}\right)$ with $\left\|\mathbf{z}^{*}\right\|_{\infty} \leq \gamma$

(iv) the constrained edge balance equations (7) have a unique solution $\psi^{*} \in \mathbb{R}^{m}$ with $\left\|\psi^{*}\right\|_{\infty} \leq \sin (\gamma)$;

(v) the unconstrained edge balance equations (8) have a unique solution $\phi^{*} \in \mathbb{R}^{m}$ with $\left\|\phi^{*}\right\|_{\infty} \leq \sin (\gamma)$.

Moreover, if one of the above equivalent conditions hold, then

$$
\mathbf{z}^{*}=B^{\top} \mathbf{x}^{*}, \quad \text { and } \quad \psi^{*}=\phi^{*}=\sin \left(B^{\top} \mathbf{x}^{*}\right) .
$$

Proof. The implications $(i) \Longrightarrow($ ii $)$ and $($ ii $) \Longrightarrow$ (iii) are easy to show.

Regarding (iii) $\Longrightarrow\left(\right.$ iv), if $\mathbf{z}^{*} \in \operatorname{Img}\left(B^{\top}\right)$ is the unique solution to the flow balance equations (6), then $\phi^{*}=\sin \left(\mathbf{z}^{*}\right) \in \mathbb{R}^{m}$ satisfies $\left\|\phi^{*}\right\|_{\infty} \leq \sin (\gamma)$ and is a solution for the edge balance equations (7). Now, we show that $\phi^{*}$ is the unique solution for the constrained edge balance equations (7) such that $\left\|\phi^{*}\right\|_{\infty} \leq \sin (\gamma)$. Suppose that $\eta^{*} \neq \phi^{*}$ is another solution of the constrained edge balance equations (7) satisfying $\left\|\eta^{*}\right\|_{\infty} \leq \sin (\gamma)$. Then, by the constrained edge balance equations (7), there exists $\mathbf{y}^{*} \in \operatorname{Img}\left(B^{\top}\right)$ such that $\mathbf{y}^{*} \neq \mathbf{z}^{*}$ and $\arcsin \left(\eta^{*}\right)=\mathbf{z}^{*}$. This implies that $\left\|\mathbf{y}^{*}\right\| \leq \gamma$ and $\mathcal{P}_{\text {cut }} \sin \left(\mathbf{y}^{*}\right)=B^{\top} L^{\dagger} \omega$. Therefore, $\mathbf{y}^{*} \in S^{G}(\gamma)$ and satisfies the flow balance equations (6). However, this is in contradiction with the facts that $\mathbf{y}^{*} \neq \mathbf{z}^{*}$ and that $\mathbf{z}^{*} \in \operatorname{Img}\left(B^{\top}\right)$ is the unique solution of the flow balance equations (6).

Regarding $(i v) \Longrightarrow(v)$, if $\psi^{*} \in \mathbb{R}^{m}$ is a solution of constrained edge balance equations (7) satisfying $\left\|\psi^{*}\right\|_{\infty} \leq \sin (\gamma)$, then

$$
\begin{aligned}
B^{\top} L^{\dagger} \omega & =\mathcal{P}_{\text {cut }} \psi^{*}, \\
\arcsin \left(\psi^{*}\right) & \in \operatorname{Img}\left(B^{\top}\right) .
\end{aligned}
$$

Because $\operatorname{Ker}\left(\mathcal{P}_{\text {cyc }}\right)=\operatorname{Img}\left(B^{\top}\right)$, the inclusion (10) implies that

$$
\mathcal{P}_{\text {cyc }} \arcsin \left(\psi^{*}\right)=0 .
$$

By adding equations (9) and (11), we obtain $B^{\top} L^{\dagger} \omega=\mathcal{P}_{\text {cut }} \psi^{*}+\mathcal{P}_{\text {cyc }} \arcsin \left(\psi^{*}\right)$. This means that $\psi^{*}$ satisfies unconstrained edge balance equations (8).

Regarding $(v) \Longrightarrow(i)$, if $\phi^{*} \in \mathbb{R}^{m}$ solves the unconstrained edge balance equations (8), then

$$
B^{\top} L^{\dagger} \omega=\mathcal{P}_{\text {cut }} \phi^{*}+\mathcal{P}_{\text {cyc }} \arcsin \left(\phi^{*}\right)
$$

Left-multiplying both sides of equations $(12)$ by $\mathcal{P}_{\text {cut }}$ and using the facts that $\mathcal{P}_{\text {cut }} B^{\top}=B^{\top}, \mathcal{P}_{\text {cut }} \mathcal{P}_{\text {cut }}=\mathcal{P}_{\text {cut }}$ and $\mathcal{P}_{\text {cut }} \mathcal{P}_{\text {cyc }}=\mathbb{D}_{m \times m}$, we obtain

$$
B^{\top} L^{\dagger} \omega=\mathcal{P}_{\text {cut }} \phi^{*} \text {. }
$$

Left-multiplying both side of the equations (12) by $\mathcal{P}_{\text {cyc }}$ we obtain

$$
\mathcal{P}_{\text {cyc }} \arcsin \left(\phi^{*}\right)=\mathbb{O}_{m} \text {. }
$$

This last equality implies that $\arcsin \left(\phi^{*}\right) \in \operatorname{Ker}\left(\mathcal{P}_{\text {cyc }}\right)=\operatorname{Img}\left(B^{\top}\right)$. Thus, there exists a vector $\mathbf{x}^{*} \in \mathbb{1}_{n}^{\perp}$ such that $\arcsin \left(\phi^{*}\right)=B^{\top} \mathbf{x}^{*}$. First, note that $\mathcal{P}_{\text {cut }} \sin \left(B^{\top} \mathbf{x}^{*}\right)=$ $B^{\top} L^{\dagger} \omega$ and, by multiplying both side of this equation by $B \mathcal{A}$, we obtain

$$
\omega=B \mathcal{A} \sin \left(B^{\top} \mathbf{x}^{*}\right)
$$


Moreover, $\left\|\phi^{*}\right\|_{\infty} \leq \gamma$. Thus, we have $\left\|\arcsin \left(\phi^{*}\right)\right\|_{\infty} \leq \gamma$ and $\left\|B^{\top} \mathbf{x}^{*}\right\|_{\infty} \leq \gamma$. This implies that $\mathbf{x}^{*} \in \mathbb{1}_{n}^{\perp}$ is a synchronization manifold for the Kuramoto model (3) in $S^{G}(\gamma)$. The uniqueness follows from [19, Theorem 10, statement (ii)].

5. Solvability of the unconstrained edge balance equations. The unconstrained edge balance equations (8) allow us to focus on a single analytic map whose inverse can be used in computing the synchronization solutions of Kuramoto model. In this section, we study the solvability of these equations and find their inverse on a suitable domain. We start with relaxing the condition $\eta \in \operatorname{Img}\left(B^{\top}\right)$ and complexifing the equations (8). This extension will allow us to use the theory of several complex variables to find the Taylor series expansion for the inversion of the complexified equations and prove the strong convergence of the Taylor series. We then restrict back to real domain and use the constrained $\eta \in \operatorname{Img}\left(B^{\top}\right)$ to find the solutions of the unconstrained edge balance equations (8). We start with some useful definitions. Given an undirected graph $G$ with cutset projection $\mathcal{P}_{\text {cut }}$ and cycle projection $\mathcal{P}_{\text {cyc }}$, define the complex edge balance map $\mathcal{F}_{\mathbb{C}}: \mathrm{D}^{\mathbb{C}}\left(\mathbb{O}_{m}, \sin (\gamma)\right) \rightarrow \mathbb{C}^{m}$ by

$$
\mathcal{F}_{\mathbb{C}}(\phi)=\mathcal{P}_{\text {cut }} \phi+\mathcal{P}_{\text {cyc }} \arcsin (\phi)
$$

and the real edge balance map $\mathcal{F}: \mathrm{D}\left(\mathbb{O}_{m}, \sin (\gamma)\right) \rightarrow \mathbb{R}^{m}$ by

$$
\mathcal{F}(\phi)=\mathcal{P}_{\text {cut }} \phi+\mathcal{P}_{\text {cyc }} \arcsin (\phi) .
$$

With this notation, the unconstrained edge balance equations (8) read $\eta=\mathcal{F}(\phi)$, together with the constraints $\|\phi\|_{\infty} \leq 1$.

Next, we define the scalar function $h: \mathbb{R}_{\geq 0} \rightarrow \mathbb{R}$ by:

$$
h(x)=(x+1) \sqrt{1-\left(\frac{x}{x+1}\right)^{2}}-x \arccos \left(\frac{x}{x+1}\right) .
$$

The graph of function $h$ on the interval $[0,20]$ is shown in Figure (1). Since $h$ is

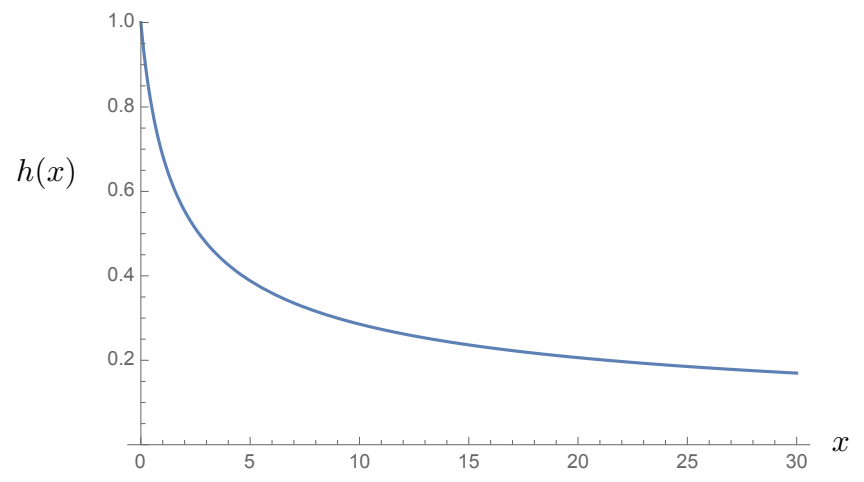

Fig. 1: The graph of the monotonically-decreasing function $h$

continuous and strictly monotonically-decreasing, its inverse exists and is denoted by $h^{-1}: \mathbb{R} \rightarrow \mathbb{R}_{\geq 0}$. Although we do not have an analytical form for $h^{-1}(y)$, it is simple to compute numerically.

We are now ready to provide an estimate on the image of the maps $\mathcal{F}_{\mathbb{C}}$ and $\mathcal{F}$ and to present a power series expansion for the inverse maps $\mathcal{F}_{\mathbb{C}}^{-1}$ and $\mathcal{F}^{-1}$ on suitable domains. 
Theorem 5 (Properties of the complex edge balance map). Consider an undirected connected graph $G$ with cutset projection $\mathcal{P}_{\text {cut }}$ and cycle projection $\mathcal{P}_{\text {cyc. }}$. Select $\eta \in \mathbb{R}^{m}$ such that $\|\eta\|_{\infty}<h\left(\left\|\mathcal{P}_{\text {cyc }}\right\|_{\infty}\right)$ and define $\gamma^{*} \in\left[0, \frac{\pi}{2}\right)$ by

$$
\gamma^{*}=\arccos \left(\frac{h^{-1}\left(\|\eta\|_{\infty}\right)}{h^{-1}\left(\|\eta\|_{\infty}\right)+1}\right) .
$$

Then the following statements holds:

(i) there exists a unique $\phi^{*} \in \mathrm{D}^{\mathbb{C}}\left(\mathbb{O}_{m}, \sin \left(\gamma^{*}\right)\right)$ such that $\mathcal{F}_{\mathbb{C}}\left(\phi^{*}\right)=\eta$; that is unconstrained edge balance equations have a unique solution;

(ii) there exists a holomorphic map $\mathcal{F}_{\mathbb{C}}^{-1}: \mathrm{D}^{\mathbb{C}}\left(\mathbb{O}_{m},\|\eta\|_{\infty}\right) \rightarrow \mathcal{F}_{\mathbb{C}}^{-1}\left(\mathrm{D}^{\mathbb{C}}\left(\mathbb{O}_{m},\|\eta\|_{\infty}\right)\right)$ such that

$$
\begin{array}{ll}
\mathcal{F}_{\mathbb{C}}^{-1} \circ \mathcal{F}_{\mathbb{C}}(\phi)=\phi, & \text { for all } \phi \in \mathcal{F}_{\mathbb{C}}^{-1}\left(\mathrm{D}^{\mathbb{C}}\left(\mathbb{O}_{m},\|\eta\|_{\infty}\right)\right), \\
\mathcal{F}_{\mathbb{C}} \circ \mathcal{F}_{\mathbb{C}}^{-1}(\xi)=\xi, & \text { for all } \xi \in \mathrm{D}^{\mathbb{C}}\left(\mathbb{O}_{m},\|\eta\|_{\infty}\right) ;
\end{array}
$$

that is the edge balance map is invertible on $\mathrm{D}^{\mathbb{C}}\left(\mathbb{O}_{m},\|\eta\|_{\infty}\right)$;

(iii) the power series

$$
\sum_{i=0}^{\infty} A_{2 i+1}(\eta)=A_{1}(\eta)+A_{3}(\eta)+A_{5}(\eta)+\ldots,
$$

converges strongly to $\mathcal{F}_{\mathbb{C}}^{-1}(\eta)$, where, for every $i \in \mathbb{Z}_{\geq 0}$, the term $A_{i}(\eta)$ is a homogeneous polynomial of order $i$ in $\eta$ defined iteratively by:

$$
\begin{aligned}
A_{1}(\eta) & =\eta \\
A_{2 i+1}(\eta) & =-\mathcal{P}_{\text {cyc }}\left(\sum_{k=1}^{i} \frac{(2 k-1) ! !}{(2 k) ! !(2 k+1)} \sum_{\begin{array}{c}
\text { odd } \\
\alpha_{1}+\cdots+\alpha_{2 k+1}=2 i+1
\end{array}} A_{\alpha_{1}}(\eta) \circ \cdots \circ A_{\alpha_{2 k+1}}(\eta)\right) .
\end{aligned}
$$

Proof. Regarding statement (i), define the map $H_{\eta}: \mathrm{D}^{\mathbb{C}}\left(\mathbb{O}_{m}, \sin \left(\gamma^{*}\right)\right) \rightarrow \mathbb{R}^{m}$ by

$$
H_{\eta}(\phi)=\eta-\mathcal{P}_{\text {cyc }}(\arcsin (\phi)-\phi) .
$$

The map $H_{\eta}$ appears from bringing all terms of the unconstrained edge equation (8) to the left hand side and adding $\phi$ to both sides.

First, we show that $H_{\eta}\left(\mathrm{D}^{\mathbb{C}}\left(\mathbb{D}_{m}, \sin \left(\gamma^{*}\right)\right)\right) \subseteq \mathrm{D}^{\mathbb{C}}\left(\mathbb{D}_{m}, \sin \left(\gamma^{*}\right)\right)$. For $\phi \in$ $\mathrm{D}^{\mathbb{C}}\left(\mathbb{O}_{m}, \sin \left(\gamma^{*}\right)\right)$, we compute

$$
\left\|H_{\eta}(\phi)\right\|_{\infty}=\left\|\eta+\mathcal{P}_{\text {cyc }}(\arcsin (\phi)-\phi)\right\|_{\infty} \leq\|\eta\|_{\infty}+\left\|\mathcal{P}_{\text {cyc }}\right\|_{\infty}\|\phi-\arcsin (\phi)\|_{\infty} .
$$

Moreover, for $\phi \in \mathrm{D}^{\mathbb{C}}\left(\mathbb{O}_{m}, \sin \left(\gamma^{*}\right)\right)$, we have $\|\phi-\arcsin (\phi)\|_{\infty} \leq \gamma^{*}-\sin \left(\gamma^{*}\right)$. These equalities imply that

$$
\begin{aligned}
\left\|H_{\eta}(\phi)\right\|_{\infty} \leq\|\eta\|_{\infty}+\left\|\mathcal{P}_{\text {cyc }}\right\|_{\infty}\left(\gamma^{*}-\sin \left(\gamma^{*}\right)\right) & \\
& \leq\|\eta\|_{\infty}+h^{-1}\left(\|\eta\|_{\infty}\right)\left(\gamma^{*}-\sin \left(\gamma^{*}\right)\right),
\end{aligned}
$$

where, for the last inequality, we used the fact that $\left\|\mathcal{P}_{\text {cyc }}\right\|_{\infty} \leq h^{-1}\left(\|\eta\|_{\infty}\right)$. By the definition of $h$, we have

$$
\begin{array}{r}
\|\eta\|_{\infty}=\left(h^{-1}\left(\|\eta\|_{\infty}\right)+1\right) \sqrt{1-\left(\frac{h^{-1}\left(\|\eta\|_{\infty}\right)}{h^{-1}\left(\|\eta\|_{\infty}\right)+1}\right)} \\
-h^{-1}\left(\|\eta\|_{\infty}\right) \arccos \left(\frac{h^{-1}\left(\|\eta\|_{\infty}\right)}{h^{-1}\left(\|\eta\|_{\infty}\right)+1}\right) .
\end{array}
$$


Noting the fact that $\arccos \left(\frac{h^{-1}\left(\|\eta\|_{\infty}\right)}{h^{-1}\left(\|\eta\|_{\infty}\right)+1}\right)=\gamma^{*}$, we obtain

$$
\|\eta\|_{\infty}=\left(h^{-1}\left(\|\eta\|_{\infty}\right)+1\right) \sin \left(\gamma^{*}\right)-h^{-1}\left(\|\eta\|_{\infty}\right) \gamma^{*} .
$$

Now, by replacing the above equation into inequality (13), we have

$$
\begin{aligned}
\left\|H_{\eta}(f)\right\|_{\infty} & \leq\|\eta\|_{\infty}+h^{-1}\left(\|\eta\|_{\infty}\right)\left(\gamma^{*}-\sin \left(\gamma^{*}\right)\right) \\
& =\left(h^{-1}\left(\|\eta\|_{\infty}\right)+1\right) \sin \left(\gamma^{*}\right)-h^{-1}\left(\|\eta\|_{\infty}\right) \gamma^{*}+h^{-1}\left(\|\eta\|_{\infty}\right)\left(\gamma^{*}-\sin \left(\gamma^{*}\right)\right) \\
& =\sin \left(\gamma^{*}\right) .
\end{aligned}
$$

Thus, by the Banach Fixed-Point Theorem, there exists a unique fixed point $\phi^{*} \in$ $\mathrm{D}^{\mathbb{C}}\left(\mathbb{O}_{m}, \sin \left(\gamma^{*}\right)\right)$ for $H_{\eta}$. By construction, this fixed element $\phi^{*} \in \mathrm{D}^{\mathbb{C}}\left(\mathbb{O}_{m}, \sin \left(\gamma^{*}\right)\right)$ satisfies

$$
\eta=\mathcal{P}_{\text {cyc }} \arcsin \left(\phi^{*}\right)+\mathcal{P}_{\text {cut }} \phi^{*}=\mathcal{F}_{\mathbb{C}}\left(\phi^{*}\right) .
$$

This completes the proof of statement (i).

Regarding statement (ii), by statement (i), for every $\eta \in \mathbb{C}^{m}$ such that $\|\eta\|_{\infty}<$ $h\left(\left\|\mathcal{P}_{\text {cyc }}\right\|_{\infty}\right)$, there exists a unique $\phi \in \mathbb{R}^{m}$ such that $\eta=\mathcal{F}_{\mathbb{C}}(\phi)$. This implies that $\mathcal{F}_{\mathbb{C}}$ has a unique inverse $\mathcal{F}_{\mathbb{C}}^{-1}: \mathcal{F}_{\mathbb{C}}\left(\mathrm{D}^{\mathbb{C}}\left(\mathbb{O}_{m},\|\eta\|_{\infty}\right)\right) \rightarrow \mathrm{D}^{\mathbb{C}}\left(\mathbb{O}_{m},\|\eta\|_{\infty}\right)$ which satisfies the equalities in statement (ii). Now we show that $\mathcal{F}_{\mathbb{C}}^{-1}$ is holomorphic on $\mathcal{F}_{\mathbb{C}}\left(\mathrm{D}^{\mathbb{C}}\left(\mathbb{O}_{m},\|\eta\|_{\infty}\right)\right)$. Note that, for every $\phi \in \mathrm{D}^{\mathbb{C}}\left(\mathbb{D}_{m},\|\eta\|_{\infty}\right)$, the derivative of the map $\mathcal{F}_{\mathbb{C}}$ at point $\phi$ is given by:

$$
D_{\phi} \mathcal{F}_{\mathbb{C}}=\mathcal{P}_{\text {cut }}+\mathcal{P}_{\text {cyc }} \operatorname{diag}\left(\frac{1}{\sqrt{1-\phi_{i}^{2}}}\right) .
$$

We first show that $D_{\phi} \mathcal{F}_{\mathbb{C}}$ is invertible. Suppose that, there exists $\mathbf{x} \in \mathbb{R}^{m}$ such that $D_{\phi} \mathcal{F}_{\mathbb{C}}(\mathbf{x})=0$. This means that $\mathcal{P}_{\text {cut }} \mathbf{x}=\mathbb{O}_{m}$ and $\mathcal{P}_{\text {cyc }} \operatorname{diag}\left(\frac{1}{\sqrt{1-\phi_{i}^{2}}}\right) \mathbf{x}=$ $\mathbb{O}_{m}$. The first equality implies that $\mathbf{x} \in \operatorname{Ker}(B \mathcal{A})$ and the second inequality implies that $\operatorname{diag}\left(\frac{1}{\sqrt{1-\phi_{i}^{2}}}\right) \mathbf{x} \in \operatorname{Img}\left(B^{\top}\right)$. Therefore, there exists $\alpha \in \mathbb{1}_{n}^{\perp}$ such that $\operatorname{diag}\left(\frac{1}{\sqrt{1-\phi_{i}^{2}}}\right) \mathbf{x}=B^{\top} \alpha$. Thus, we get

$$
B \mathcal{A} \operatorname{diag}\left(\sqrt{1-\phi_{i}^{2}}\right) B^{\top} \alpha=B \mathcal{A} \mathbf{x}=\mathbb{O}_{n} .
$$

Moreover, $\mathcal{A} \operatorname{diag}\left(\sqrt{1-\phi_{i}^{2}}\right)$ is a diagonal matrix with positive diagonal elements. Therefore, equations (14) implies that $\alpha \in \operatorname{span}\left\{\mathbb{1}_{n}\right\}$ and as a result $\mathbf{x}=$ $\operatorname{diag}\left(\sqrt{1-\phi_{i}^{2}}\right) B^{\top} \alpha=\mathbb{O}_{n}$. This proves that the derivative $D_{\phi} \mathcal{F}_{\mathbb{C}}$ is invertible. Now, by the Inverse Function Theorem [1, Theorem 2.5.2], the maps $\mathcal{F}_{\mathbb{C}}$ and $\mathcal{F}_{\mathbb{C}}^{-1}$ are locally holomorphic and therefore they are holomorphic on their domains. This completes the proof of statement (ii).

Regarding statement (iii), we first find the formal power series representation for $\mathcal{F}_{\mathbb{C}}^{-1}$. Suppose that $\sum_{i=1}^{\infty} A_{i}(\xi)$ is the formal power series for $\mathcal{F}_{\mathbb{C}}^{-1}$. Then we have

$$
\mathcal{P}_{\text {cyc }} \arcsin \left(\mathcal{F}_{\mathbb{C}}^{-1}(\eta)\right)+\mathcal{P}_{\text {cut }} \mathcal{F}_{\mathbb{C}}^{-1}(\eta)=\eta, \quad \text { for all } \eta \in \mathbb{R}^{m} .
$$

By replacing the power series $\sum_{i=1}^{\infty} A_{i}(\xi)$ for $\mathcal{F}_{\mathbb{C}}^{-1}$ and using the power series expansion of arcsin, we obtain

$$
\mathcal{P}_{\text {cyc }}\left(\sum_{k=1}^{\infty} \frac{(2 k-1) ! !}{(2 k) ! !(2 k+1)}\left(\sum_{i=1}^{\infty} A_{i}(\eta)\right)^{\circ(2 k-1)}\right)+\mathcal{P}_{\text {cut }} \sum_{i=1}^{\infty} A_{i}(\eta)=\eta .
$$


By equating the same order terms on the both side of equation (15) and using the fact that $\mathcal{P}_{\text {cyc }}+\mathcal{P}_{\text {cut }}=I_{m}$, we obtain that $A_{1}(\eta)=\eta$ and $A_{2 i}(\eta)=\mathbb{O}_{m}$, for every $i \in \mathbb{Z}_{\geq 0}$. Simple book-keeping shows that the recursive formula in statement (iii) holds for the odd terms in the power series.

Finally, we prove that the formal power series $\sum_{i=1}^{\infty} A_{2 i+1}(\eta)$ converges on the domain $\mathrm{D}^{\mathbb{C}}\left(\mathbb{O}_{m}, \eta\right)$. Note that statement (ii) implies that the map $\mathcal{F}_{\mathbb{C}}^{-1}: \mathrm{D}^{\mathbb{C}}\left(\mathbb{O}_{m}, \eta\right) \rightarrow$ $\mathcal{F}_{\mathbb{C}}^{-1}\left(\mathrm{D}^{\mathbb{C}}\left(\mathbb{O}_{m}, \eta\right)\right)$ is holomorphic and and that the set $\mathrm{D}^{\mathbb{C}}\left(\mathbb{O}_{m}, \eta\right)$ is a Reinhardt domain. Therefore, [16, Theorem 2.4.5] implies that the power series converges strongly on the domain $\mathrm{D}^{\mathbb{C}}\left(\mathbb{O}_{m}, \eta\right)$.

REMARK 1 (Properties of the real edge balance map). A similar result as Theorem 5 holds for the real edge balance map $\mathcal{F}$ by replacing the complex variables by their real counterparts. The proof is straightforward by restricting the results in Theorem 5 to the real Euclidean space.

6. Inverse Taylor expansion for Kuramoto model. In this section we study the synchronization of the Kuramoto model (3) by applying the results on the unconstrained edge balance equations (8) from Theorem 5 in the previous section.

Theorem 6 (Inverse Taylor expansion). Consider the Kuramoto model (3) with undirected connected graph $G$, weighted cutset projection $\mathcal{P}_{\text {cut }}$, and weighted cycle projection $\mathcal{P}_{\text {cyc }}$. Given frequencies $\omega \in \mathbb{1}_{n}^{\perp}$ satisfying

$$
\left\|B^{\top} L^{\dagger} \omega\right\|_{\infty}<h\left(\left\|\mathcal{P}_{\text {cyc }}\right\|_{\infty}\right)
$$

define $\gamma^{*} \in\left[0, \frac{\pi}{2}\right)$ by

$$
\gamma^{*}=\arccos \left(\frac{h^{-1}\left(\left\|B^{\top} L^{\dagger} \omega\right\|_{\infty}\right)}{h^{-1}\left(\left\|B^{\top} L^{\dagger} \omega\right\|_{\infty}\right)+1}\right)
$$

Then the following statements hold:

(i) there exists a unique locally stable synchronization manifold $\mathbf{x}^{*}$ in $S^{G}\left(\gamma^{*}\right)$; and

(ii) the power series

$$
\sum_{i=0}^{\infty} A_{2 i+1}\left(B^{\top} L^{\dagger} \omega\right)=A_{1}\left(B^{\top} L^{\dagger} \omega\right)+A_{3}\left(B^{\top} L^{\dagger} \omega\right)+\ldots
$$

converges strongly to $\sin \left(B^{\top} \mathbf{x}^{*}\right)$ where, for every $i \in \mathbb{Z}_{\geq 0}$, the term $A_{i}(\eta)$ is a homogeneous polynomial of order $i$ in $\eta$ defined iteratively as in Theorem 5 (iii).

This theorem is an immediate application of Theorem 4 on the equivalent transcriptions and of Theorem 5 on the properties of the maps $\mathcal{F}_{\mathbb{C}}$ and $\mathcal{F}$.

Proof of Theorem 6. Regarding statement (i), we use (the real version of) Theorem 5(i) with $\eta=B^{\top} L^{\dagger} \omega$. Since $\left\|B^{\top} L^{\dagger} \omega\right\|_{\infty}<h\left(\left\|\mathcal{P}_{\text {cyc }}\right\|_{\infty}\right)$. Therefore, there exists a unique $\phi^{*} \in \mathrm{D}\left(\mathbb{D}_{m}, \sin \left(\gamma^{*}\right)\right)$ such that $\mathcal{F}(\phi)=B^{\top} L^{\dagger} \omega$. This means that

$$
B^{\top} L^{\dagger} \omega=\mathcal{P}_{\text {cyc }} \arcsin \left(\phi^{*}\right)+\mathcal{P}_{\text {cut }} \phi^{*}
$$

Since $\operatorname{Img}\left(B^{\top}\right)=\operatorname{Img}\left(\mathcal{P}_{\text {cut }}\right)=\operatorname{Ker}\left(\mathcal{P}_{\text {cyc }}\right)$, we obtain $\mathcal{P}_{\text {cut }} \phi^{*}=B^{\top} L^{\dagger} \omega$ and $\arcsin (\phi)^{*} \in \operatorname{Img}\left(B^{\top}\right)$. The result follows by the equivalence of parts (i) and (iv) in Theorem 4.

Regarding statement (ii), the result follows from (the complex version of) Theorem 5 (iii). 
Some remarks are in order.

REMARK 2 (Power series expansion for $\sin \left(B^{\top} \mathbf{x}^{*}\right)$ ).

(i) It is instructive to apply the iterative procedure in Theorem 6(ii) to compute the first four odd terms in the power series (16) where $\eta=B^{\top} L^{\dagger} \omega$ :

$$
\begin{aligned}
& A_{1}(\eta)=\eta \\
& A_{3}(\eta)=-\mathcal{P}_{\text {cyc }}\left(\frac{1}{6} \eta^{\circ 3}\right) \\
& A_{5}(\eta)=-\mathcal{P}_{\text {cyc }}\left(\frac{1}{12} A_{3}(\eta) \circ \eta^{\circ 2}+\frac{3}{40} \eta^{\circ 5}\right) \\
& A_{7}(\eta)=-\mathcal{P}_{\text {cyc }}\left(\frac{5}{112} \eta^{\circ 7}+\frac{3}{8} A_{3}(\eta) \circ \eta^{\circ 4}+\frac{1}{2}\left(A_{3}(\eta)\right)^{\circ 2} \circ \eta+\frac{1}{2} A_{5}(\eta) \circ \eta^{\circ 2}\right) .
\end{aligned}
$$

The iterative procedure in Theorem 6(ii) is amenable to implementation on a mathematical software manipulation system; we report its implementation in Mathematica code in Algorithm 1 in Appendix A.

(ii) If $\mathcal{P}_{\text {cyc }}$ and $\operatorname{diag}(\eta)$ commute, then, for every $i \in \mathbb{Z}_{>0}$ :

$$
A_{2 i+1}(\eta)=-\frac{(2 i-1) ! !}{(2 i) ! !(2 i+1)} \mathcal{P}_{\text {cyc }}(\eta)^{\circ(2 i+1)} .
$$

For example, if the graph $G$ is acyclic, then $\mathcal{P}_{\text {cyc }}=\mathbb{D}_{n \times n}$ and, therefore, $\mathcal{P}_{\text {cyc }}$ and $\operatorname{diag}(\eta)$ commute. Thus, for acyclic graphs, we have

$$
\begin{aligned}
A_{1}(\eta) & =\eta, \\
A_{2 i+1}(\eta) & =0, \quad \text { for all } i \in \mathbb{Z}_{\geq 0}
\end{aligned}
$$

so that $\sin \left(B^{\top} \mathbf{x}^{*}\right)=B^{\top} L^{\dagger} \omega$. Therefore, the Kuramoto model (3) on an acyclic graph has a unique locally stable synchronization manifold inside $S^{G}(\gamma)$ if and only if $\left\|B^{\top} L^{\dagger} \omega\right\|_{\infty} \leq \sin (\gamma)$. Moreover, if this condition holds, then the synchronization manifold is given by

$$
\mathbf{x}^{*}=L^{\dagger} B \mathcal{A} \arcsin \left(B^{\top} L^{\dagger} \omega\right) .
$$

This result is known for example as [12, Theorem 2 (Supporting Information)].

(iii) While for acyclic graphs we have $\mathcal{P}_{\text {cyc }}=\mathbb{O}_{n \times n}$, the matrix $\mathcal{P}_{\text {cyc }}$ is non-zero and idempotent for cyclic graphs and it satisfies $\left\|\mathcal{P}_{\text {cyc }}\right\|_{\infty} \geq 1$. The jump from $\left\|\mathcal{P}_{\text {cyc }}\right\|_{\infty}$ equals 0 to values greater than or equal to 1 can be attributed to the discontinuity of the projection matrix $\mathcal{P}_{\text {cyc }}$ with respect to edge weights of the graph. The following example shows that the infinity norm of the projection matrix $\mathcal{P}_{\text {cyc }}$ is, in general, a discontinuous function of the weights of the graphs. Consider the family of 3-cycle graph $\{G(\epsilon)\}_{\epsilon \geq 0}$ with the node set $V=\{1,2,3\}$, the edge set $\mathcal{E}=\{(1,2),(1,3),(2,3)\}$, and the adjacency matrix $A(\epsilon) \in \mathbb{R}^{3 \times 3}$ given by

$$
A(\epsilon)=\left(\begin{array}{lll}
0 & 1 & 1 \\
1 & 0 & \epsilon \\
1 & \epsilon & 0
\end{array}\right)
$$


Then, for every $\epsilon>0$, one can show that

$$
\mathcal{P}_{\text {cyc }}(\epsilon)=I_{3}-B^{\top} L^{\dagger} B \mathcal{A}=\left(\begin{array}{ccc}
\epsilon & \epsilon & \epsilon \\
\epsilon & \epsilon & \epsilon \\
1-2 \epsilon & 1-2 \epsilon & 1-2 \epsilon
\end{array}\right) .
$$

This implies that $\lim _{\epsilon \rightarrow 0^{+}}\left\|\mathcal{P}_{\text {cyc }}(\epsilon)\right\|_{\infty}=3$. However, the graph $G(0)$ is acyclic and therefore we have $\mathcal{P}_{\text {cyc }}(0)=\mathbb{O}_{3 \times 3}$. Thus, $\lim _{\epsilon \rightarrow 0^{+}}\left\|\mathcal{P}_{\text {cyc }}\right\|_{\infty} \neq$ $\left\|\mathcal{P}_{\text {cyc }}(0)\right\|_{\infty}$. This implies that the function $\epsilon \mapsto\left\|\mathcal{P}_{\text {cyc }}(\epsilon)\right\|_{\infty}$ is not continuous at $\epsilon=0$.

In the rest of this section, we use the power series (16) to propose a family of statistically-accurate approximate tests that are much less conservative than proven sufficient conditions for existence of a unique synchronization solution inside $S^{G}(\gamma)$ for $\gamma \in[0, \pi / 2)$. Our approximate tests estimate the solution of the unconstrained edge balance equations (8) and check that all elements of the estimate are less than or equal to $\sin (\gamma)$. For more insight into these approximate tests, recall from Theorem 6(ii) that $\phi$, the solution for unconstrained edge balance equations (8) is given by

$$
\phi=B^{\top} L^{\dagger} \omega+A_{3}\left(B^{\top} L^{\dagger} \omega\right)+A_{5}\left(B^{\top} L^{\dagger} \omega\right)+\ldots
$$

There already exists a first order approximate synchronization test, introduced by reference [12], which truncates the series, given above, after the first order term. By approximating $\phi$ with $\phi \approx B^{\top} L^{\dagger} \omega \in \operatorname{Img}\left(B^{\top}\right)$, we write

$$
\left\|B^{\top} L^{\dagger} \omega\right\|_{\infty} \leq \sin (\gamma)
$$

By substituting the third order power series expansion for the edge variable $\phi \in \mathbb{R}^{m}$ of the Kuramoto model in Theorem 6(ii) into equation (7), we can also write the third order approximate synchronization test as

$$
\left\|B^{\top} L^{\dagger} \omega+\frac{1}{6} \mathcal{P}_{\text {cyc }}\left(B^{\top} L^{\dagger} \omega\right)^{\circ 3}\right\|_{\infty} \leq \sin (\gamma)
$$

In summary we propose a family of higher order approximate tests as follows.

DeFinition 7. For $\gamma \in[0, \pi / 2)$ and $k \in 2 \mathbb{Z}_{\geq 0}+1$, the $k$ th order approximate test for existence of a unique solution in $S^{G}(\gamma)$ is defined by

$$
\left\|\sum_{i=0}^{(k-1) / 2} A_{2 i+1}\left(B^{\top} L^{\dagger} \omega\right)\right\|_{\infty} \leq \sin (\gamma) .
$$

7. Numerical Experiments. In this section we illustrate the usefulness of the Taylor series expansion given by Theorem 6. First, for large IEEE test cases, we illustrate the accuracy of the truncated series for approximating synchronized solutions of (3). In addition, we present results showing the sharpness of the approximate tests (ATk) for existence of a synchronization manifold (7) on several IEEE test cases and random networks.

7.1. Accuracy of the Taylor series: approximating the synchronization manifold. Here we evaluate the accuracy of the truncated power series in Theorem 6(ii) for approximating the synchronization manifold. We consider both IEEE test cases and random networks to evaluate these measures of accuracy. 
The general numerical setting for the IEEE test cases is as follows. Each IEEE test case can be described by a connected undirected graph $G$ with the nodal admittance matrix $Y \in \mathbb{C}^{n \times n}$. The set of nodes in $G$ are partitioned into load buses $\mathcal{N}_{1}$ and generator buses $\mathcal{N}_{2}$. The power demand (resp. power injection) at node $i \in \mathcal{N}_{1}$ (resp. $i \in \mathcal{N}_{2}$ ) is denoted by $P_{i} . \quad V_{i}$ and $\theta_{i}$ are the voltage magnitude and phase angle at node $i \in \mathcal{N}_{1} \cup \mathcal{N}_{2}$. For every IEEE test case, we study the following Kuramoto synchronization manifold equation

$$
P_{i}=\sum_{j \in \mathcal{N}_{1} \cup \mathcal{N}_{2}} a_{i j} \sin \left(\theta_{i}-\theta_{j}\right), \quad \text { for all } i \in \mathcal{N}_{1} \cup \mathcal{N}_{2},
$$

where $a_{i j}=a_{j i}=V_{i} V_{j} \Im\left(Y_{i j}\right)>0$. The equations (17) are exactly the lossless active $\mathrm{AC}$ power flow equations for the network. Note that in order to study equations (17), we need to apply some modifications to the IEEE test cases. First, the admittance matrix $Y \in j \mathbb{R}^{n \times n}$ is purely inductive with no shunt admittances $Y_{i i}=0$. If the IEEE test case has branch resistances or shunt admittances, then they are removed. Second, we assume that all the nodes in the IEEE test case are $P V$ nodes; this assumption is reasonable since the active power injection and output voltage of generators are known. For the loads, we use MATPOWER [44] to solve the coupled AC power flow balance to obtain their terminal voltage $V_{i}$. Lastly, for every $i \in\{1, \ldots, n\}$, we set $P_{i}=K P_{i}^{\text {nom }}$ for some $K \in \mathbb{R}_{\geq 0}$ where $P_{i}^{\text {nom }}$ is the nominal injections given by each test case. Starting with $K=0$, we increase $K$ by $5 \times 10^{-3}$ at each step. MATLAB's fsolve is used to solve equations (17) for $\theta_{\mathrm{fsolve}}^{*}$ at each $K$. The scalar $K$ is increased until whichever situation occurs first: $\left\|B^{\top} \theta_{\mathrm{fsolve}}^{*}\right\|_{\infty}$ reaches $\pi / 2$ or fsolve does not converge to a solution.

To evaluate the accuracy of the truncated Taylor series with $k$ terms, we define the absolute error denoted by $S_{k}$ by

$$
S_{k}=\left\|\sin \left(B^{\top} \theta_{\mathrm{fsolve}}^{*}\right)-\sum_{i=0}^{k} A_{2 i+1}\left(B^{\top} L^{\dagger} p_{\mathrm{sd}}\right)\right\|_{\infty},
$$

where $p_{\text {sd }}=\left[P_{1}, \ldots, P_{n}\right]^{\top}$ is the balanced supply/demand vector, $L=B \mathcal{A} B^{\top}$, and $\mathcal{A}$ is the diagonal weight matrix matrix with diagonal elements $\left\{a_{i j}\right\}_{(i, j) \in \mathcal{E}}$. The errors $S_{k}$ for IEEE 300 and Pegase 1354 are shown in Figure 2.
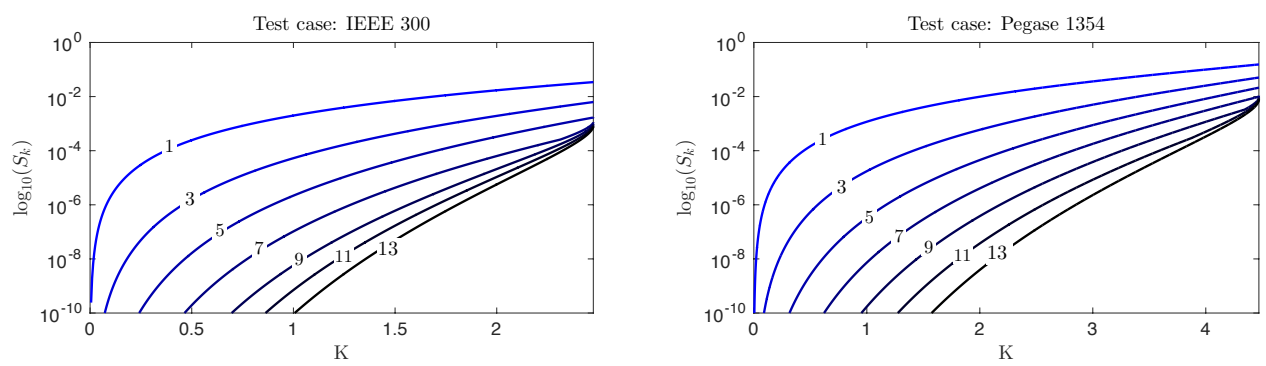

Fig. 2: Comparison of the absolute errors of the sine of the phase differences approximated with the truncated Taylor series in Theorem 6(ii) for all odd orders up to 13. The absolute error is calculated with equation (18), using the solution found with fsolve as the true value. 
Summary evaluation. Figure 2 shows that, for the IEEE test cases, the error of truncated Taylor series for computing the synchronized solutions of the Kuramoto model (17) decreases exponentially with the order of the truncations and increases as we approach the threshold of synchronization. For IEEE 300 and Pegase 1354 with the nominal power injections, the error of approximating the synchronized manifold with 5 th order or higher truncated series is smaller than $10^{-6}$.

7.2. Accuracy of the Taylor series: approximating the critical coupling. In this section, we compare the approximate synchronization test (ATk) with the existing tests in the literature and evaluate the accuracy of these approximate tests. We consider both IEEE test cases and random networks to evaluate these measures of accuracy.

For IEEE test cases, we use the same simulation setup given earlier in Section 7.1. Denote the critical coupling of equation (17) by $K_{\mathrm{C}}$, that is, let $K_{\mathrm{C}}$ be the smallest scaling factor such that $\left\|B^{\top} \theta_{\text {fsolve }}^{*}\right\|_{\infty}$ reaches $\pi / 2$ or fsolve does not converge to a solution (whichever occurs first). Let $K_{\mathrm{T}}$ denote the smallest scaling factor for which a particular synchronization test fails. Then we denote the critical ratio by $K_{\mathrm{T}} / K_{\mathrm{C}}$; this percentage is a measure of the accuracy of the given test. The conditions are checked with a $10^{-6}$ tolerance. Table 1 compares the accuracy of the approximate test with existing sufficient conditions for synchronization:

The first two columns contain the critical ratio of two known tests from the literature (T1) and (T2) from [11, Theorem 7.2] and [19, Theorem 16] respectively:

$$
\begin{aligned}
\lambda_{2}(L) & >\lambda_{\text {critical }} \triangleq\left\|B^{\top} p_{\text {sd }}\right\|_{2}, \\
\left\|B^{\top} L^{\dagger} p_{\text {sd }}\right\|_{\infty} & \leq g\left(\left\|\mathcal{P}_{\text {cut }}\right\|_{\infty}\right) .
\end{aligned}
$$

Note that test (T2) is a sufficient condition for existence of a synchronization manifold in $S^{G}\left(\gamma^{*}\right)$ where $\gamma^{*}=\arccos \left(\frac{\left\|\mathcal{P}_{\text {cut }}\right\|_{\infty}-1}{\left\|\mathcal{P}_{\text {cut }}\right\|_{\infty}+1}\right) \in[0, \pi / 2]$ and $g(x)=\frac{y(x)+\sin (y(x))}{2}-$ $\left.x \frac{y(x)-\sin (y(x))}{2}\right|_{y(x)=\arccos \left(\frac{x-1}{x+1}\right)}$.

The third column contains the new sufficient test (T0) proposed in this paper.

The last four columns contains the critical ratio for the family of approximate tests $(\mathrm{AT} k)$ of order $1,3,5$ and 7.

\begin{tabular}{|l|c|c|c|c|c|c|c|}
\hline \multirow{3}{*}{ Test Case } & \multicolumn{7}{|c|}{ Critical ratio $K_{\mathrm{T}} / K_{\mathrm{C}}$} \\
\cline { 2 - 8 } & $\begin{array}{c}\lambda_{2} \text { test } \\
(\mathrm{T} 1)[1]\end{array}$ & $\begin{array}{c}\text { D-norm } \\
\text { test } \\
(\mathrm{T} 2)[19]\end{array}$ & $\begin{array}{c}\text { New test } \\
(\mathrm{T} 0)\end{array}$ & $\begin{array}{c}\text { Approx test } \\
k=1 \\
(\mathrm{AT} 1)[12]\end{array}$ & $\begin{array}{c}\text { Approx test } \\
k=3 \\
(\mathrm{AT} k)\end{array}$ & $\begin{array}{c}\text { Approx test } \\
k=5 \\
(\mathrm{AT} k)\end{array}$ & $\begin{array}{c}\text { Approx test } \\
k=7 \\
(\mathrm{AT} k)\end{array}$ \\
\hline IEEE 118 & $0.23 \%$ & $43.76 \%$ & $29.91 \%$ & $86.12 \%$ & $90.80 \%$ & $93.10 \%$ & $94.45 \%$ \\
IEEE 300 & $0.02 \%$ & $40.45 \%$ & $27.25 \%$ & $99.64 \%$ & $99.80 \%$ & $99.84 \%$ & $99.88 \%$ \\
Pegase 1354 & $0.04 \%$ & $34.04 \%$ & $23.94 \%$ & $89.02 \%$ & $97.58 \%$ & $99.61 \%$ & $99.66 \%$ \\
Polish 2383 & $0.03 \%$ & $29.49 \%$ & $20.60 \%$ & $84.53 \%$ & $90.62 \%$ & $92.60 \%$ & $93.95 \%$ \\
\hline
\end{tabular}

IEEE test cases from [5] and Pegase test case from [20].

Table 1: Comparison of the conservativeness of various sufficient conditions with approximate synchronizations tests applied to IEEE test cases in the domain $S^{G}(\pi / 2)$.

Summary evaluation. Table 1 shows that, for IEEE test cases with scaled nominal power injections, the following statements holds:

(i) the accuracy of the approximate tests (ATk) increases with the order of the tests; 
(ii) for $\gamma=\frac{\pi}{2}$, the fifth and seventh order approximate tests (ATk) improves the accuracy given by the 1 st order approximate test $\left\|B^{\top} L^{\dagger} \omega\right\|_{\infty} \leq 1$ [12] by up to $9 \%$;

(iii) for $\gamma=\frac{\pi}{2}$, the fifth and seventh order approximate tests (ATk) improves the best-known sufficient synchronization test in the literature [19] by up to $50 \%$.

Next, we consider random graph models with randomly generated natural frequencies. We setup the numerical analysis to assess the correctness of the family of approximate tests (ATk) as follows. Consider a nominal unweighted random networks $\{G, \omega\}$, where $G$ is a connected undirected graph with $n=80$ nodes chosen from a parametrized family of random graph models RGM and $\omega \in \mathbb{1}_{n}^{\perp}$ are natural frequencies chosen randomly form sampling distribution SD. Then we study the synchronization of the Kuramoto model with uniform coupling gain $K \in \mathbb{R}_{>0}$,

$$
\dot{\theta}=\omega-K B \sin \left(B^{\top} \theta\right) .
$$

The random graph models RGM and the sampling distributions SD are given as follows:

(i) Network topology: For the network topology, we consider three types of random graph models RGM. The random graph models we consider are: (i) Erdős-Rényi random graph with probability $p$ of an edge existing [7], (ii) Random Geometric graph model with sampling region $(0,1]^{2} \subset \mathbb{R}^{2}$ and connectivity radius $p$ [7], and (iii) Watts-Strogatz small world model network with initial coupling to the 2 nearest neighbors and rewiring probability $p$ of an edge existing [43]. If there exists an edge, then the coupling weight is $a_{i j}=a_{j i}=1$. If the graph is not connected, then it is thrown out and a new random graph is generated.

(ii) Natural frequencies: We consider two types of sampling distributions SD. $n=80$ random numbers are sampled from either a (i) uniform distribution on the interval $(-1,1)$ or (ii) bipolar distribution $\{-1,+1\}$ to obtain $q_{i}$ for $i \in\{1, \ldots, n\}$. Then to ensure that $\mathrm{t}$ he natural frequencies satisfy $\omega \in \mathbb{1}_{n}^{\perp}$, we take $\omega_{i}=q_{i}-\sum_{i=1}^{n} q_{i} / n$.

(iii) Parametric realizations: We consider combinations of parameters $(R G M, p, S D)$ : the three random graph models, 15 edge connectivity parameters on the interval $p \in(0,1)$, and two sampling distributions.

For each parametric realization in (iii), we generate 100 nominal models of $\{G, \omega \in$ $\left.\mathbb{1}_{n}^{\perp}\right\}$. For each nominal case, we find the critical coupling, denoted by $K_{\mathrm{C}}$, and the smallest coupling where the approximate test fails (7), denoted by $K_{\mathrm{T}}$, for orders $\{1,3,5,7\} . K_{\mathrm{C}}$ is found iteratively with MATLAB's fsolve. We define the normalized critical coupling ratio of each random case by $K_{\mathrm{C}} / K_{\mathrm{T}}$. The numerically determined values are found with an accuracy of $10^{-3}$. Each data point in Figure 3 corresponds to the mean of $K_{\mathrm{C}} / K_{\mathrm{T}}$ over 100 nominal cases of the same parametric realization.

Summary evaluation. Figure (3) illustrates that, for random graph models with random natural frequency from bipolar and uniform distribution and $\gamma=\frac{\pi}{2}$, the accuracy of the approximate test (ATk) consistently improves. In particular, the fifth and seventh order approximate tests (ATk) improves the accuracy of the 1st order approximate test $\left\|B^{\top} L^{\dagger} \omega\right\|_{\infty} \leq 1[12]$ by up to $30 \%$.

7.3. Computational cost of approximating the synchronization manifold. Consider a connected graph $G$ with $m$ edges, $n$ nodes, and no self-loops. Table 2 shows the order of the number of operations associated with different methods for approximating the synchronization manifold for the sparse and dense graphs. 


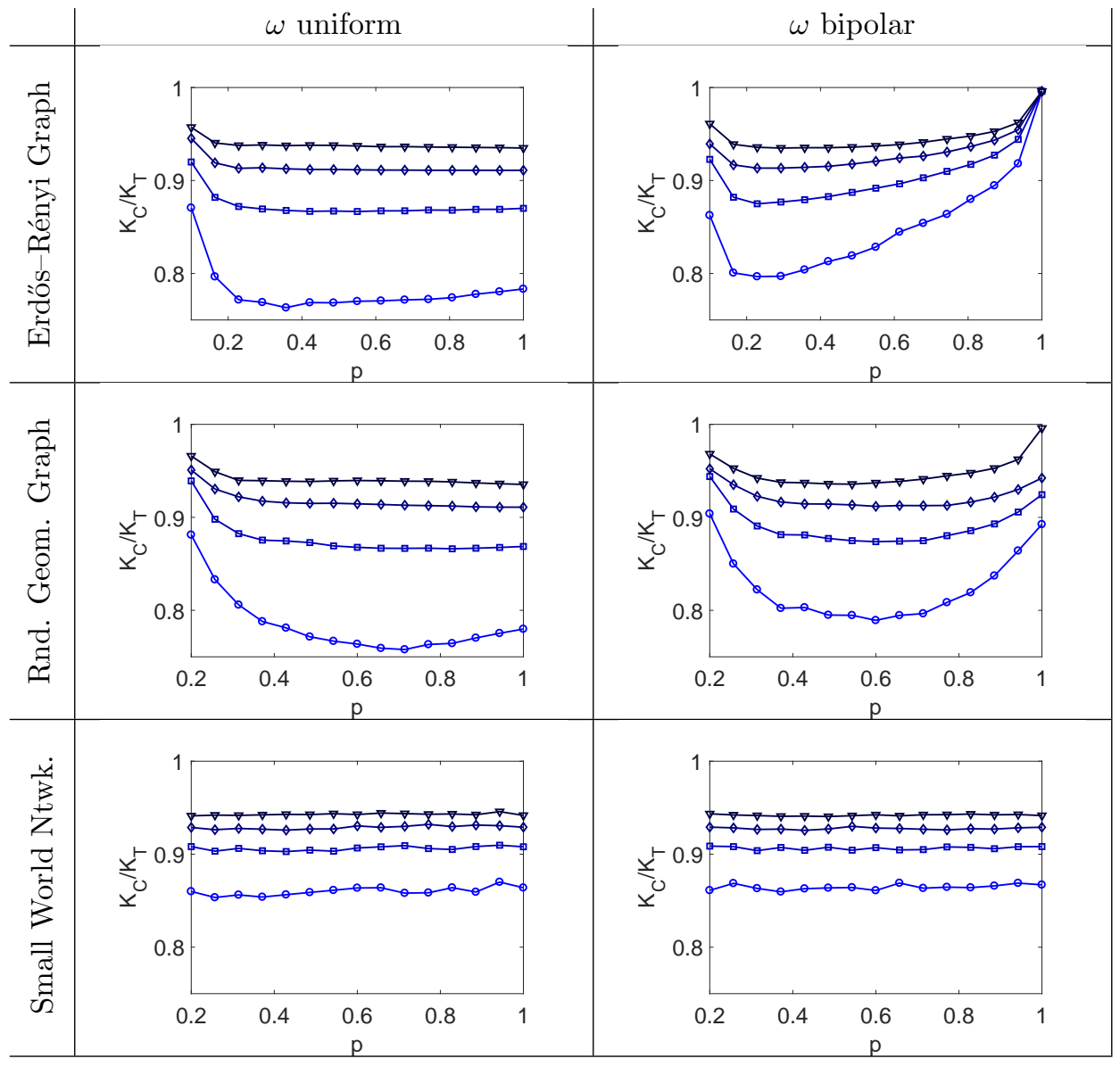

$\rightarrow$ Order $1 \rightarrow$ Order $3 \rightarrow$ Order $5 \rightarrow$ Order 7

Fig. 3: Each data point is the critical ratio $K_{\mathrm{C}} / K_{\mathrm{T}}$ averaged over 100 random graphs with $n=80$ nodes and in the domain $S^{G}(\pi / 2) . K_{\mathrm{C}} / K_{\mathrm{T}}$ measures the accuracy of the approximate synchronization tests $(\mathrm{AT} k) . K_{\mathrm{C}}$ is the smallest coupling gain such that there exists a solution to the Kuramoto model. $K_{\mathrm{T}}$ is an approximation of $K_{\mathrm{C}}$, estimated using the approximate test (ATk) derived from Theorem 6(ii) for orders $k=1,3,5,7$.

For random graph models, we compare the computational time of three different methods for approximating the synchronization manifold of the Kuramoto model: (i) the series approximation of the analytical solution from Theorem 6(ii), (ii) Newton-Raphson method, and (iii) MATLAB's fsolve.

For the simulation setup, we consider the random network $\{G, \omega\}$ with $n \geq 2$ nodes, $\omega_{i} \in(-\alpha, \alpha)$ for $i \in\{1, \ldots, n\}$, and number of edges $m$ depending on the coupling parameter $p \in(0,1)$. The following lists the random graph parameters:

(i) Network topology: To construct the random graph, the Erdős-Rényi random graph model was used with probability $p$ of an edge existing. If the graph is not connected, then it is thrown out and a new random graph is generated. 


\begin{tabular}{|c|c|c|c|}
\hline Method & General & $\begin{array}{c}\text { Sparse Graphs } \\
\mathcal{O}(n)=\mathcal{O}(m)\end{array}$ & $\begin{array}{c}\text { Dense Graphs } \\
\mathcal{O}\left(n^{2}\right)=\mathcal{O}(m)\end{array}$ \\
\hline Precomputation & $\mathcal{O}\left(m^{2} n\right)$ & $\mathcal{O}\left(n^{3}\right)$ & $\mathcal{O}\left(n^{5}\right)$ \\
Series, 5th order* & $\mathcal{O}\left(2 m^{2}\right)$ & $\mathcal{O}\left(2 n^{2}\right)$ & $\mathcal{O}\left(2 n^{4}\right)$ \\
Series, 7th order* & $\mathcal{O}\left(3 m^{2}\right)$ & $\mathcal{O}\left(3 n^{2}\right)$ & $\mathcal{O}\left(3 n^{4}\right)$ \\
Newton-Raphson & $\mathcal{O}\left(m n^{2}\right)$ & $\mathcal{O}\left(n^{3}\right)$ & $\mathcal{O}\left(n^{4}\right)$ \\
\hline
\end{tabular}

* Denotes that the method precomputes the terms $B^{\top} L^{\dagger}, L^{\dagger} B \mathcal{A}$ and

$\mathcal{P}_{\text {cyc. }}$. The computation complexity of these terms are found in the "Precomputation" row.

The computational complexity of $L^{\dagger}$ for $L \in \mathbb{R}^{n \times n}$ is $\mathcal{O}\left(n^{3}\right)$.

Table 2: Comparison of number of operations required for computing the truncated series and Newton-Raphson.

(ii) Coupling weights: Each edge is given a random coupling weight, $a_{i j}=a_{j i}>0$, sampled on the uniform distribution interval $(0,10)$.

(iii) Natural frequencies: $n$ random numbers are sampled from a uniform distribution on the interval $(-\alpha, \alpha)$ to obtain $q_{i}$ for $i \in\{1, \ldots, n\}$. Then to ensure that the natural frequencies satisfy $\omega \in \mathbb{1}_{n}^{\perp}$, we take $\omega_{i}=q_{i}-\sum_{i=1}^{n} q_{i} / n . \alpha$ is chosen to be sufficiently small so that the MATLAB fsolve converges to a solution of the Kuramoto model (3).

(iv) Parametric realizations: We consider random network parametrization $(n, p, \alpha)$ with combinations of $n=\{10,20,30,60,120\}$ and $p=$ $\{0.2,0.4,0.6,0.8\}$.

For each parametrization, we generate 3000 nominal graphs and 20 natural frequency vectors for each random graph. The results of the execution time for various methods are shown in Figure 4 where each point is the computational time for a particular method averaged over 3000 graphs and 20 natural frequency vectors $\omega \in \mathbb{1}_{n}^{\perp}$ per graph. The computation time for the series approximation is the total time to complete the calculations for 1 random nominal graph with 20 different natural frequency vectors. This time does not include the computation time for the precomputed terms listed in Table 2. The initial guess for Newton-Raphson and fsolve is $B^{\top} L^{\dagger} \omega$.

Summary evaluation. Figure 4 show that the computation time for the truncated power series increases with density of the random graphs. Moreover, the truncated series are more efficient than Newton-Raphson method for small random graphs, while they are only comparable to Newton-Raphson method for large random graphs.

For IEEE test cases, we compare the computational time of three different methods for calculating the synchronization manifold: (i) the series approximation of the analytical solution from Theorem 6(ii), (ii) Newton-Raphson method, and (iii) MATLAB's fsolve. The setting for the IEEE test cases are the same as the one given in Section 7.1. In this setup we do not precompute any terms and consider one graph topology with its nominal power injections. We use each method to solve for the synchronization manifold $\left[\theta^{*}\right]$, and average the computation time over 10 trials.

Summary evaluation. The results are found in Table 3 show that, for IEEE test cases, the series approximations are computationally comparable to Newton-Raphson.

8. Conclusion. This paper proposes a novel equivalent characterization of the equilibrium equation for the Kuramoto coupled oscillator; we refer to this characterization as to the unconstrained edge balance equation. Using this characterization, 


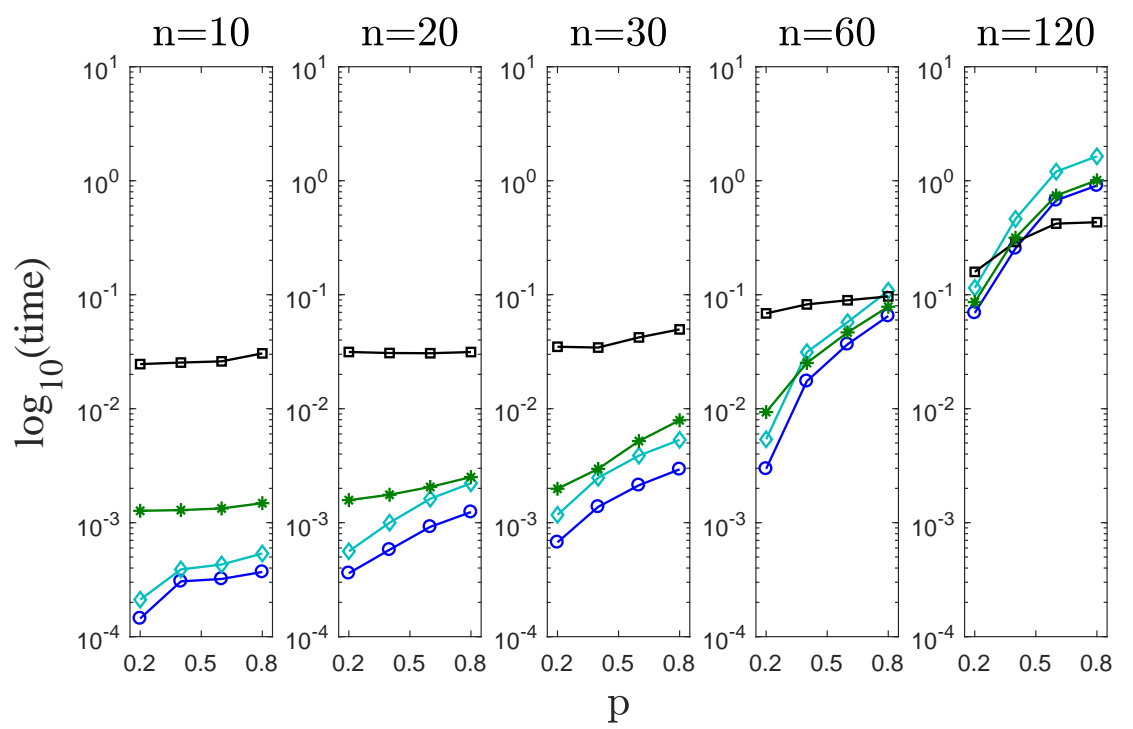

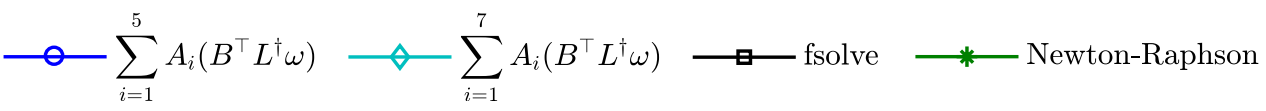

Fig. 4: Comparison of computation times for 5th order truncated series, the 7th order truncated series, MATLAB's fsolve, and Newton-Raphson for random graphs, where $p$ is the probability of an edge existing for Erdős-Rényi graphs. The computation time is how long it takes the various methods to compute the solutions of the unconstrained edge balance equations, for 20 randomly generated natural frequency vector given one randomly generated graph. If a random natural frequency vector does not give a solution, it is thrown out and a new vector is generated. Certain values are precomputed for each graph, but the precomputation time is not included in the graph. Each data point is averaged over 3000 Erdős-Rényi random graphs.

\begin{tabular}{|c|c|c|c|}
\hline Test Case & fsolve / NR & Ord. 5 / NR & Ord. 7 / NR \\
\hline IEEE 118 & 4.2072 & 0.4511 & 0.4539 \\
IEEE 300 & 2.6501 & 0.7546 & 0.7539 \\
Pegase 1354 & 1.2825 & 0.8582 & 0.8633 \\
Polish 2383 & 1.1279 & 0.9559 & 0.9583 \\
\hline
\end{tabular}

Table 3: Computational times of MATLAB's fsolve, fifth order series (Ord. 5), and 7th order series (Ord. 7) normalized by the computational time for Newton-Raphson (NR) for IEEE test cases. The computation time is how long it takes the various methods to compute the solution of the unconstrained edge balance equations, averaged over 10 trials. Certain values are precomputed for each graph, but the precomputation times are not included.

we propose a Taylor series expansion for the synchronization manifold of the $\mathrm{Ku}$ ramoto network and a recursive formula to symbolically compute all the terms in the Taylor series. We then use the truncated Taylor series as a tool to (i) find sharp ap- 
proximation for the synchronization manifold and (ii) estimate the onset of frequency synchronization. Our numerical simulations illustrate the accuracy and computational efficiency of this method on various classes of random graphs and IEEE test cases. As future directions, it may be instructive to employ this series expansion method to study frequency synchronization in networks consisting of other important oscillators, such as FitzHugh-Nagumo systems. Additionally, it may be viable to adopt the series expansion approach to tackle more general nonlinear network flow problems, such as the coupled power flow equations and optimal power flow problems.

\section{Appendix A. Mathematica Code.}

In this appendix, we present an implementation of a Mathematica algorithm to compute the coefficient of the power series expansion given in Theorem 6(ii).

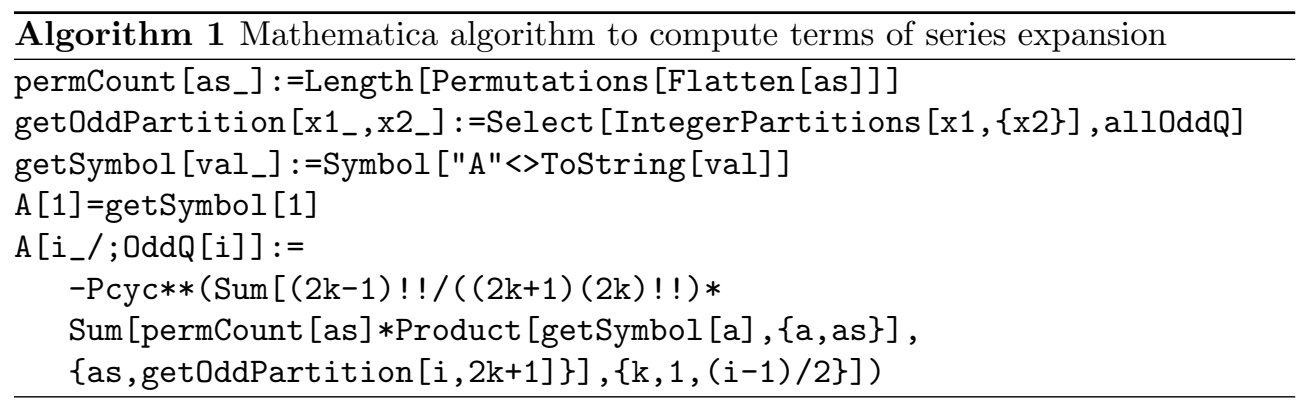

It is worth mentioning that the required computations increase exponentially with the order of the terms. Specifically, computing the $(2 k+1)$ th order coefficient of the power series requires finding all the odd-integer partitions of $2 k+1$.

\section{REFERENCES}

[1] R. Abraham, J. E. Marsden, and T. S. Ratiu. Manifolds, Tensor Analysis, and Applications, volume 75 of Applied Mathematical Sciences. Springer, 2 edition, 1988.

[2] J. A. Acebrón, L. L. Bonilla, C. J. P. Vicente, F. Ritort, and R. Spigler. The Kuramoto model: A simple paradigm for synchronization phenomena. Reviews of Modern Physics, 77(1):137-185, 2005. doi:10.1103/RevModPhys.77.137.

[3] D. Aeyels and J. A. Rogge. Existence of partial entrainment and stability of phase locking behavior of coupled oscillators. Progress of Theoretical Physics, 112(6):921-942, 2004. doi:10.1143/PTP.112.921.

[4] N. Ainsworth and S. Grijalva. A structure-preserving model and sufficient condition for frequency synchronization of lossless droop inverter-based AC networks. IEEE Transactions on Power Systems, 28(4):4310-4319, 2013. doi:10.1109/TPWRS.2013.2257887.

[5] A. B. Birchfield, T. Xu, K. M. Gegner, K. S. Shetye, and T. J. Overbye. Grid structural characteristics as validation criteria for synthetic networks. IEEE Transactions on Power Systems, 32(4):3258-3265, 2017. doi:10.1109/TPWRS.2016.2616385.

[6] F. Bullo. Lectures on Network Systems. CreateSpace, 1 edition, 2018. With contributions by J. Cortés, F. Dörfler, and S. Martínez. URL: http://motion.me.ucsb.edu/book-lns.

[7] G. Chen, X. Wang, and X. Li. Fundamentals of Complex Networks: Models, Structures and Dynamics. John Wiley \& Sons, 2015. doi:10.1002/9781118718124.

[8] N. Chopra and M. W. Spong. On exponential synchronization of Kuramoto oscillators. IEEE Transactions on Automatic Control, 54(2):353-357, 2009. doi:10.1109/TAC. 2008. 2007884.

[9] F. Dörfler and F. Bullo. On the critical coupling for Kuramoto oscillators. SIAM Journal on Applied Dynamical Systems, 10(3):1070-1099, 2011. doi:10.1137/10081530x.

[10] F. Dörfler and F. Bullo. Synchronization and transient stability in power networks and nonuniform Kuramoto oscillators. SIAM Journal on Control and Optimization, 50(3):16161642, 2012. doi:10.1137/110851584. 
[11] F. Dörfler and F. Bullo. Synchronization in complex networks of phase oscillators: A survey. Automatica, 50(6):1539-1564, 2014. doi:10.1016/j .automatica.2014.04.012.

[12] F. Dörfler, M. Chertkov, and F. Bullo. Synchronization in complex oscillator networks and smart grids. Proceedings of the National Academy of Sciences, 110(6):2005-2010, 2013. doi:10.1073/pnas.1212134110.

[13] G. B. Ermentrout and N. Kopell. Multiple pulse interactions and averaging in systems of coupled neural oscillators. Journal of Mathematical Biology, 29(3):195-217, 1991. doi: 10.1007/BF00160535.

[14] A. F. Glimn and G. W. Stagg. Automatic calculation of load flows. Transactions of the American Institute of Electrical Engineers. Part III: Power Apparatus and Systems, 76(3):817825, 1957. doi:0.1109/AIEEPAS.1957.4499665.

[15] D. J. Hill and G. Chen. Power systems as dynamic networks. In IEEE Int. Symposium on Circuits and Systems, pages 722-725, Kos, Greece, May 2006. doi:10.1109/ISCAS. 2006. 1692687.

[16] L. Hörmander. An Introduction to Complex Analysis in Several Variables, volume 7 of NorthHolland Mathematical Library. North-Holland Publishing Co, third edition, 1990.

[17] E. Y. Huang, S. Jafarpour, and F. Bullo. Synchronization of coupled oscillators: The Taylor expansion of the inverse Kuramoto map. In IEEE Conf. on Decision and Control, Miami, USA, December 2018. To appear. URL: https://arxiv.org/abs/1803.08277.

[18] A. Jadbabaie, N. Motee, and M. Barahona. On the stability of the Kuramoto model of coupled nonlinear oscillators. In American Control Conference, pages 4296-4301, Boston, USA, June 2004. doi:10.23919/ACC.2004.1383983.

[19] S. Jafarpour and F. Bullo. Synchronization of Kuramoto oscillators via cutset projections. IEEE Transactions on Automatic Control, November 2017. Submitted. URL: https:// arxiv.org/abs/1711.03711.

[20] C. Josz, S. Fliscounakis, J. Maeght, and P. Panciatici. AC power flow data in MATPOWER and QCQP format: iTesla, RTE snapshots, and PEGASE, March 2016. URL: https: //arxiv.org/abs/1603.01533.

[21] D. J. Klein, P. Lee, K. A. Morgansen, and T. Javidi. Integration of communication and control using discrete time Kuramoto models for multivehicle coordination over broadcast networks. IEEE Journal on Selected Areas in Communications, 26(4):695-705, 2008. doi: 10.1109/JSAC. 2008.080511.

[22] Y. Kuramoto. Self-entrainment of a population of coupled non-linear oscillators. In H. Araki, editor, Int. Symposium on Mathematical Problems in Theoretical Physics, volume 39 of Lecture Notes in Physics, pages 420-422. Springer, 1975. doi:10.1007/BFb0013365.

[23] R. Kyng and S. Sachdeva. Approximate Gaussian elimination for Laplacians - Fast, sparse, and simple. In IEEE Annual Symposium on Foundations of Computer Science, pages 573-582, New Brunswick, USA, 2016. doi:10.1109/FOCS.2016.68.

[24] D. Mehta, N. S. Daleo, F. Dörfler, and J. D. Hauenstein. Algebraic geometrization of the Kuramoto model: Equilibria and stability analysis. Chaos: An Interdisciplinary Journal of Nonlinear Science, 25(5):053103, 2015. doi:10.1063/1.4919696.

[25] R. E. Mirollo and S. H. Strogatz. The spectrum of the locked state for the Kuramoto model of coupled oscillators. Physica D: Nonlinear Phenomena, 205(1-4):249-266, 2005. doi: $10.1016 / j \cdot$ physd.2005.01.017.

[26] D. K. Molzahn, F. Dörfler, H. Sandberg, S. H. Low, S. Chakrabarti, R. Baldick, and J. Lavaei. A survey of distributed optimization and control algorithms for electric power systems. IEEE Transactions on Smart Grid, 8(6):2941-2962, 2017. doi:10.1109/TSG.2017.2720471.

[27] J. A. Momoh, R. Adapa, and M. E. El-Hawary. A review of selected optimal power flow literature to 1993. I. Nonlinear and quadratic programming approaches. IEEE Transactions on Power Systems, 14(1):96-104, 1999. doi:10.1109/59.744492.

[28] J. A. Momoh, M. E. El-Hawary, and R. Adapa. A review of selected optimal power flow literature to 1993. II. Newton, linear programming and interior point methods. IEEE Transactions on Power Systems, 14(1):105-111, 1999. doi:10.1109/59.744495.

[29] J. Munkres. Topology. Prentice Hall, 2000.

[30] S. Rao, Y. Feng, D. J. Tylavsky, and J. K. Subramanian. The holomorphic embedding method applied to the power-flow problem. IEEE Transactions on Power Systems, 31(5):38163828, 2016. doi:10.1109/TPWRS.2015.2503423.

[31] R. Sepulchre, D. A. Paley, and N. E. Leonard. Stabilization of planar collective motion: Allto-all communication. IEEE Transactions on Automatic Control, 52(5):811-824, 2007. doi:10.1109/TAC. 2007.898077.

[32] O. Simeone, U. Spagnolini, Y. Bar-Ness, and S. H. Strogatz. Distributed synchronization in wireless networks. IEEE Signal Processing Magazine, 25(5):81-97, 2008. doi:10.1109/ 
MSP. 2008.926661.

[33] D. A. Spielman and S.-H. Teng. Nearly linear time algorithms for preconditioning and solving symmetric, diagonally dominant linear systems. SIAM Journal on Matrix Analysis and Applications, 35(3):835-885, 2014. doi:10.1137/090771430.

[34] B. Stott and O. Alsac. Fast decoupled load flow. IEEE Transactions on Power Apparatus and Systems, 93(3):859-869, 1974. doi:10.1109/TPAS.1974.293985.

[35] B. Stott, J. Jardim, and O. Alsac. DC power flow revisited. IEEE Transactions on Power Systems, 24(3):1290-1300, 2009. doi:10.1109/TPWRS.2009.2021235.

[36] C. J. Tavora and O. J. M. Smith. Stability analysis of power systems. IEEE Transactions on Power Apparatus and Systems, 91(3):1138-1144, 1972. doi:10.1109/TPAS.1972.293470.

[37] W. F. Tinney and C. E. Hart. Power flow solution by Newton's method. IEEE Transactions on Power Apparatus and Systems, PAS-86(11):1449-1460, 1967. doi:10.1109/TPAS. 1967. 291823.

[38] A. Trias. The holomorphic embedding load flow method. In IEEE Power \& Energy Society General Meeting, pages 1-8, 2012. doi:10.1109/PESGM.2012.6344759.

[39] M. Verwoerd and O. Mason. Global phase-locking in finite populations of phase-coupled oscillators. SIAM Journal on Applied Dynamical Systems, 7(1):134-160, 2008. doi: 10.1137/070686858.

[40] N. K. Vishnoi. $L x=b$, Laplacian solvers and their algorithmic applications. Theoretical Computer Science, 8(1-2):1-141, 2013. doi:10.1561/0400000054.

[41] C. Wang, N. Rubido, C Grebogi, and M. S. Baptista. Approximate solution for frequency synchronization in a finite-size Kuramoto model. Physical Review E, 92(6):062808, 2015. doi:10.1103/PhysRevE.92.062808.

[42] Y. Wang and F. J. Doyle III. Exponential synchronization rate of Kuramoto oscillators in the presence of a pacemaker. IEEE Transactions on Automatic Control, 58(4):989-994, 2013. doi:10.1109/TAC.2012.2215772.

[43] D. J. Watts and S. H. Strogatz. Collective dynamics of 'small-world' networks. Nature, 393:440442, 1998. doi:10.1038/30918.

[44] R. D. Zimmerman, C. E. Murillo-Sánchez, and R. J. Thomas. MATPOWER: Steady-state operations, planning, and analysis tools for power systems research and education. IEEE Transactions on Power Systems, 26(1):12-19, 2011. doi:10.1109/TPWRS.2010.2051168. 